\title{
Resident fibroblast lineages mediate pressure overload-induced cardiac fibrosis
}

\author{
Thomas Moore-Morris, ${ }^{1}$ Nuno Guimarães-Camboa, ${ }^{1}$ Indroneal Banerjee, ${ }^{2}$ Alexander C. Zambon, ${ }^{3}$ \\ Tatiana Kisseleva, ${ }^{2}$ Aurélie Velayoudon, ${ }^{1}$ William B. Stallcup, ${ }^{4}$ Yusu Gu, ${ }^{2}$ Nancy D. Dalton, ${ }^{2}$ \\ Marta Cedenilla, ${ }^{1}$ Rafael Gomez-Amaro, ${ }^{1}$ Bin Zhou, ${ }^{5}$ David A. Brenner, ${ }^{2}$ \\ Kirk L. Peterson, ${ }^{2}$ Ju Chen, ${ }^{2}$ and Sylvia M. Evans ${ }^{1,2}$
}

\begin{abstract}
1Skaggs School of Pharmacy, ${ }^{2}$ Department of Medicine, and ${ }^{3}$ Department of Pharmacology, UCSD, La Jolla, California, USA. ${ }^{4}$ Sanford-Burnham Medical Research Institute, La Jolla, California, USA. ${ }^{5}$ Department of Genetics, Pediatrics and Medicine, Albert Einstein College of Medicine of Yeshiva University, New York, New York, USA.
\end{abstract}

\begin{abstract}
Activation and accumulation of cardiac fibroblasts, which result in excessive extracellular matrix deposition and consequent mechanical stiffness, myocyte uncoupling, and ischemia, are key contributors to heart failure progression. Recently, endothelial-to-mesenchymal transition (EndoMT) and the recruitment of circulating hematopoietic progenitors to the heart have been reported to generate substantial numbers of cardiac fibroblasts in response to pressure overload-induced injury; therefore, these processes are widely considered to be promising therapeutic targets. Here, using multiple independent murine Cre lines and a collagen1a1-GFP fusion reporter, which specifically labels fibroblasts, we found that following pressure overload, fibroblasts were not derived from hematopoietic cells, EndoMT, or epicardial epithelial-to-mesenchymal transition. Instead, pressure overload promoted comparable proliferation and activation of two resident fibroblast lineages, including a previously described epicardial population and a population of endothelial origin. Together, these data present a paradigm for the origins of cardiac fibroblasts during development and in fibrosis. Furthermore, these data indicate that therapeutic strategies for reducing pathogenic cardiac fibroblasts should shift from targeting presumptive EndoMT or infiltrating hematopoietically derived fibroblasts, toward common pathways upregulated in two endogenous fibroblast populations.
\end{abstract}

\section{Introduction}

Heart failure is the primary cause of adult mortality in the developed world $(1,2)$. A key component of heart failure is excessive extracellular matrix deposition by cardiac fibroblasts (CFs) that leads to fibrosis and pathological remodeling of myocardium (3). During development, fibroblasts derive from epicardial cells that undergo an epithelial-to-mesenchymal transition (EMT) $(4,5)$. However, in pathological contexts, such as hypertrophy, a large proportion of CFs are thought to derive from nonepicardial lineages. Indeed, one study has reported that up to $70 \%$ of CFs in pressure overload-induced cardiac lesions were derived from pathologically induced endothelial-to-mesenchymal transition (EndoMT) (6). Diabetes has also been reported to induce pathological cardiac EndoMT (7). Furthermore, cells of hematopoietic origin have been reported to be a major source of CFs in heart disease $(6,8)$. Finally, in the context of myocardial infarction, activation of adult epicardium has been shown to generate fibroblasts (9). Thus, these processes responsible for generating CFs in disease settings have been put forward as important therapeutic targets $(6,7)$. In particular, targeting EndoMT has been lauded as having potential to preserve the vasculature while reducing fibroblast numbers.

Current views on the origins of CF lineages during development and in disease have been established without the use of specific markers or systematic quantitative analysis of fibroblast lineages present in heart. Markers used to identify fibroblasts, such as fibroblast-specific protein 1 (FSP1, also known as S100A4), label only a subset of fibroblasts and are expressed by several other cell

Conflict of interest: The authors have declared that no conflict of interest exists. Citation for this article: J Clin Invest. 2014;124(7):2921-2934. doi:10.1172/JCI74783. types, including endothelial and immune cells $(6,10,11)$. Previous evidence for EndoMT was based on results using lineage tracing with Tie1-Cre, which labels both endothelial and immune lineages, and FSP1, as a marker of fibroblasts (6). The finding that FSP1 labels immune cells suggests the importance of revisiting the issue of EndoMT using more specific fibroblast markers. Identification and characterization of specific CF markers are critical to understanding of mechanisms generating these cells in fibrotic conditions. Furthermore, a clear picture of CF lineage development would provide an important basis from which to investigate mechanisms responsible for fibroblast accumulation during fibrosis in adult heart. This is critical in order to identify strategies for much needed antifibrotic therapies.

In the present study, we reexamined the origins of CFs during development and in fibrosis by performing genetic lineage tracing of CFs using markers that we demonstrated are specific to CFs, including a GFP reporter driven by a collagen $1 \mathrm{a} 1$ enhancer (12). Our studies revealed that CFs include two main lineages of epicardial and endothelial origins. Importantly, we showed that fibroblast accumulation associated with pressure overload hypertrophy resulted from activation and proliferation of these resident lineages and not EndoMT, hematopoietic progenitor recruitment, or epicardial activation. Using multiple Cre drivers, we showed that a previously overlooked fibroblast lineage of endothelial origin likely derives from endocardium at the time of endocardial cushion development. Transcriptional profiling of the two distinct fibroblast lineages revealed that they responded similarly to pressure overload. Taken together, our studies demonstrated that activation of resident fibroblast lineages should be targeted to alleviate fibrosis. 
A
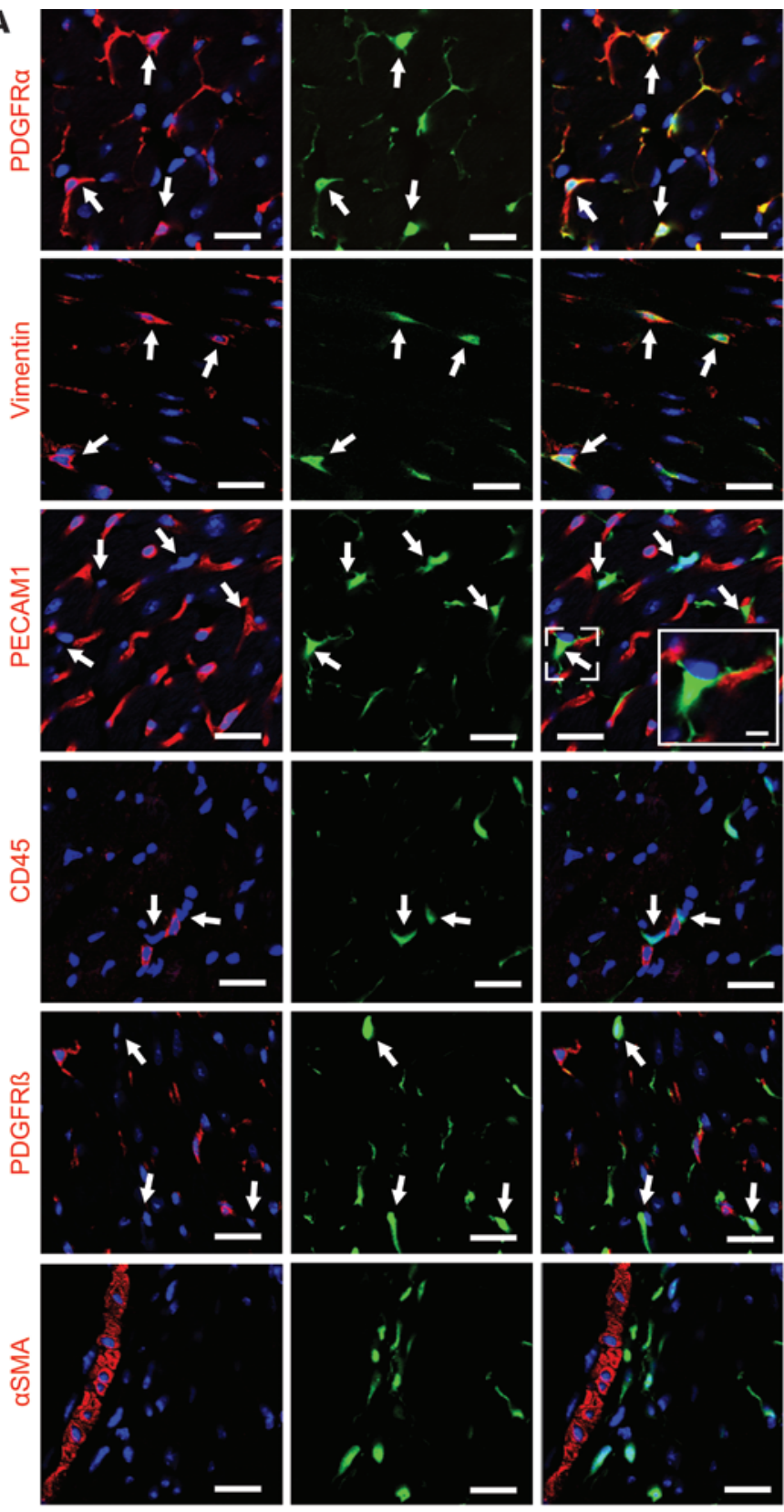

DAPI

Collagen1a1-GFP

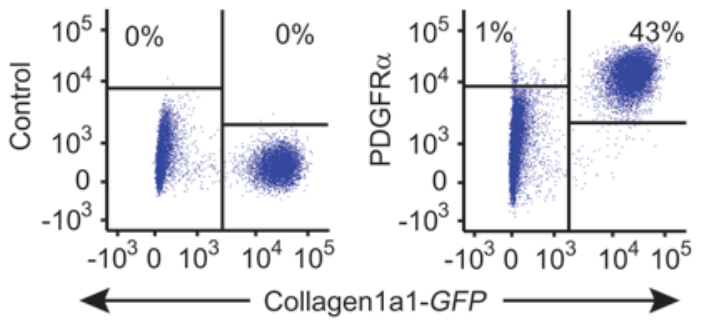

C
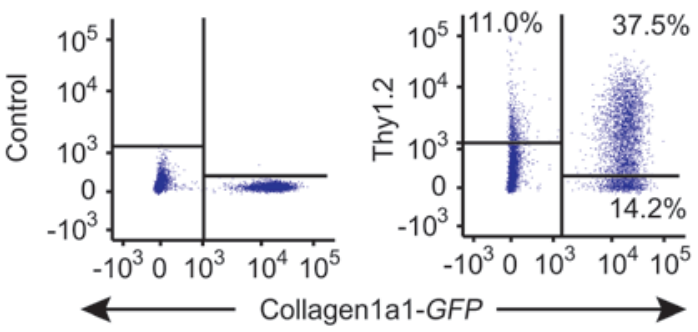

D

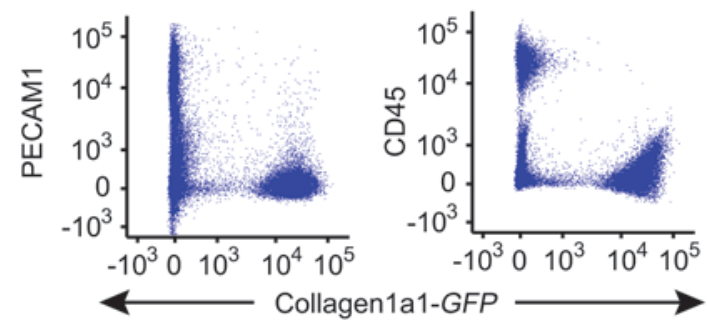

$\mathbf{E}$

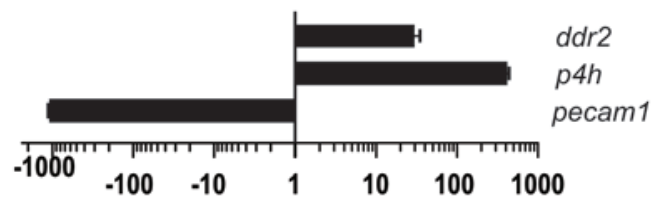

mRNA fold enrichment in Collagen1a1-GFP+ cells versus PECAM1+ endothelium

$\mathbf{F}$

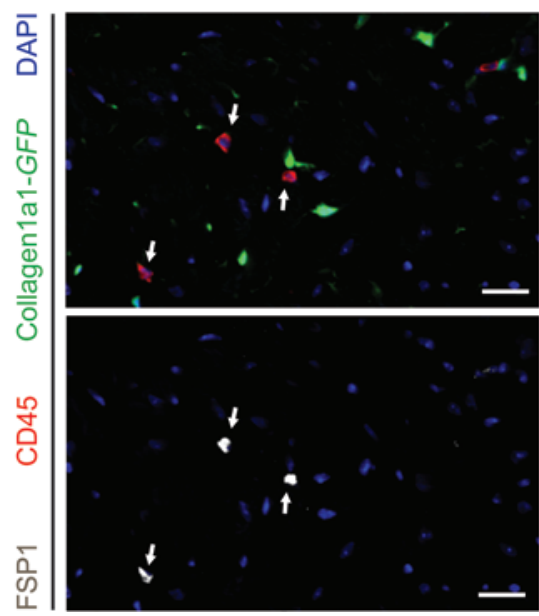

Figure 1

CF markers in adult murine myocardium. (A) Confocal analysis of ventricular myocardium shows that collagen1a1-GFP labels CFs (arrows) that expressed the mesenchymal marker PDGFR $\alpha$ and vimentin. Collagen1a1-GFP+ fibroblasts were negative for markers of endothelium (PECAM1), blood lineages (CD45), pericytes (PDGFR $\beta$ ), and smooth muscle $(\alpha S M A)$. (B) Flow cytometry analysis of dissociated LV and IVS, showing overlap of collagen1a1-GFP and PDGFR $\alpha$ signals. (C) Flow cytometry analysis of LV and IVS, showing that collagen1a1-GFP cells are mostly, but not all, Thy1.2 positive. (D) Collagen1a1-GFP+ cells were PECAM1- and CD45- . (E) Quantitative real-time PCR showing fold enrichment of DDR2, prolyl-4-hydroxylase (p4h), and PECAM1 in flow cytometry-sorted collagen1a1-GFP+ and Pecam1 ${ }^{+}$cells $(n=3$ hearts, average \pm SEM). (F) Confocal images showing colocalization of FSP1 CD45+ leukocytes (arrows) but not with collagen1a1-GFP fibroblasts. Images are representative of at least 3 hearts. Scale bars: $20 \mu \mathrm{m} ; 5 \mu \mathrm{m}$ (inset). 


\section{Results}

Markers for CFs in normal and failing heart. Identifying markers expressed by CFs both at baseline and in disease settings has been problematic $(11,13)$. Fibroblasts are defined as interstitial mesenchymal-type cells that secrete extracellular matrix constituents, notably collagen type I (3). Hence, to identify CFs, we investigated the utility of a transgenic GFP reporter mouse line driven by a collagen $1 \mathrm{a} 1$ enhancer (12). Confocal/FACS analysis of adult left ventricular (LV) myocardium and interventricular septum (IVS) (5-week-old males) demonstrated that collagen1a1-GFP cells were all vimentin ${ }^{+}$and PDGFR $\alpha^{+}$(Figure 1, A and B). PDGFR $\alpha$ is a mesenchymal marker recently shown to be expressed by CFs $(14,15)$. Approximately two-thirds of collagen1a1-GFP ${ }^{+}$cells expressed Thy1, which is known to label subsets of fibroblasts as well as immune and endothelial lineages (Figure 1C and ref. 16). Further confocal and FACS analysis revealed that all interstitial and perivascular collagen1a1-GFP+ cells were $\alpha \mathrm{SMA}^{-}$(smooth muscle cell marker), PDGFR $\beta^{\mathrm{lo} /-}$ (pericyte marker), PECAM1- (pan-endothelial marker), and CD45(pan-leukocyte marker) (Figure 1, A and D). We also found RNA expression of fibroblast markers discoidin domain receptor-2 (DDR2) and prolyl-4-hydroxylase to be enriched in FACS-sorted collagen1a1-GFP+ fibroblasts compared with PECAM1+ endothelium (Figure 1E). In agreement with previous reports using FSP1 as a fibroblast marker $(6,17)$, we found FSP1 ${ }^{+}$cells to be rare in normal adult myocardium. The few FSP $1^{+}$cells, however, did not colocalize with collagen1a1-GFP or PDGFR $\alpha$. Instead, they colocalized with CD45 (Figure 1F), consistent with a recent report showing that FSP1 labels immune cells in heart (11).

We then subjected mice to transaortic constriction (TAC) (18) and observed significant hypertrophy and fibrosis at day 7 and day 28 after surgery (Supplemental Table 1 and Supplemental Figure 1; supplemental material available online with this article; doi:10.1172/JCI74783DS1). Analysis of adjacent sections showed that pathologic fibrotic lesions, as revealed with immunostaining against collagen type I and trichrome staining, corresponded strikingly to areas of pronounced collagen $1 \mathrm{a} 1-G F P^{+} \mathrm{CF}$ accumulation (Figure 2A). Fibrosis is found in spatially distinct locations; interstitial fibrosis occurs around myocytes, while perivascular fibrosis occurs in the vicinity of large coronary vessels. Therefore, we felt it important to examine both interstitial and perivascular fibroblasts in fibrotic areas in a quantitative manner. Approximately $30 \%$ of all cells within interstitial lesions were CD $45^{+}$immune cells, and $49 \%$ were fibroblasts marked by collagen1a1-GFP (Figure 2, B and C). Notably, FSP1 ${ }^{+}$cells in interstitial fibrotic areas were almost exclusively CD45+ leukocytes (Figure 2, B and C). In perivascular lesions, we observed FSP1 expression in a few collagen 1a1-GFP+ fibroblasts, although FSP1 expression was relatively weak when compared with its expression in adjacent $\mathrm{CD} 45^{+}$leukocytes (Supplemental Figure 2, A and B). In sham-operated controls, perivascular fibroblasts were $\mathrm{FSP} 1^{-}$, but many perivascular $\mathrm{CD}_{4} 5^{+}$leukocytes were FSP $1^{+}$(Supplemental Figure 2C). $\alpha \mathrm{SMA}$ expression was evident in approximately $15 \%$ of collagen $1 \mathrm{a} 1-G F P^{+}$ fibroblasts in interstitial fibrotic regions but was absent in perivascular fibroblasts (Figure 2, D and E, and Supplemental Figure 2, D and E). Similar observations were made following 28 days of TAC (Supplemental Figure 2F). The foregoing demonstrated potential pitfalls in using either FSP1 or $\alpha$ SMA as comprehensive markers for fibroblasts within fibrotic lesions in pressure overload. In contrast, confocal and flow cytometry analysis demonstrated that
collagen1a1-GFP and PDGFR $\alpha$ comprehensively and specifically marked CFs (Figure 2F and Supplemental Figure 3). Thus, we used these as markers to determine the lineage origin of fibroblasts within fibrotic lesions following pressure overload.

CF lineages in normal and hypertrophic heart. The widely accepted and potentially clinically relevant concept of pathological cardiac EndoMT is based on a study that reported the emergence of a Tie1Cre-labeled FSP1+ "fibroblast" population after, but not before, pressure overload (6). Our findings on the pitfalls of using FSP1 as a fibroblast marker (Figure $1 \mathrm{~F}$ and Figure 2, B and C) prompted us to reevaluate the origins of activated CFs during pressure overload.

Although all CFs are thought to derive from epicardium during development (12), no study has directly addressed this important issue. We generated Wt1-Cre ${ }^{+/-}$collagen1a1-GFP ${ }^{+/-}$Rosa-td $T^{+/-}$mice, in which, as expected, all fibroblasts were labeled in right ventricles (RVs) and atria (Supplemental Figure 4A and refs. 19, 20). Unexpectedly, however, in the LV free wall (LVFW) and the IVS, only $92 \% \pm 2 \%$ and $30 \% \pm 6 \%$ of fibroblasts were labeled by Wt1-Cre, respectively (average \pm SD of 10 fields, from 2 hearts) (Figure 3A). Many myocytes were found to be labeled in the IVS, as previously reported with another Wt1-Cre driver (21). A similar pattern of CF labeling was observed with Tbx18-Cre (5), another epicardial-specific Cre driver (Supplemental Figure 4, B and C).

Early in development, highly orchestrated EndoMT of a subset of endocardial cells gives rise to fibroblasts within the atrioventricular (AV) cardiac cushions $(22,23)$. Whether this lineage expands beyond the cushions has not yet been investigated. To address this question, we performed Tie2-Cre lineage tracing, which was previously used to demonstrate endocardial contribution to valve mesenchyme (22). As expected, recombination occurred in endothelium throughout the myocardium. Interestingly, we found a spatial distribution of Tie2-Cre-labeled fibroblasts within adult myocardium that was complementary to that of Wt1-Cre labeling, with $64 \% \pm 8 \%$ and $12 \% \pm 3 \%$ of fibroblasts being labeled by Tie2-Cre within the IVS and LVFW, respectively (average \pm SD of 10 fields, from 2 hearts) (Figure 3B).

Flow cytometry analysis of myocardium (LV and IVS) after removing valve tissue and epicardium showed that overall the majority of fibroblasts $(85 \% \pm 2 \%$, mean $\pm \mathrm{SD}, n=3)$ were Wt1-Cre lineage traced, but that a significant number $(18 \% \pm 2 \%$ mean \pm $\mathrm{SD}, n=3$ ) were Tie2-Cre lineage traced (Figure 3, C and D). Tie2-Cre labels both endothelial and blood lineages; however, no fibroblasts were labeled by hematopoietic-specific Vav-Cre (Figure 3, $\mathrm{C}$ and D), suggesting that Tie2-Cre lineage-traced fibroblasts were of endothelial origin. Importantly, approximately $95 \%(n=3)$ of all fibroblasts were lineage traced in Tie2-Cre $e^{+/} \mathrm{Wt}^{-}-\mathrm{Cre}^{+/-}$collagen1a1-GFP $P^{+/-}$Rosa-td $T^{+/-}$mice, demonstrating that Tie2-Cre and Wt1-Cre labeled two largely distinct populations, which together comprised the vast majority of CFs (Figure 3, C and D).

Following pressure overload, although fibroblast numbers were visibly increased, the distribution of Tie2-Cre- and Wt1-Cre-labeled fibroblast lineages in interstitial and perivascular areas remained comparable to that of sham-operated controls. Indeed, Wt1Cre- and Tie2-Cre-labeled fibroblasts were predominant in either the LVFW or IVS, respectively (Figure 4A). In perivascular areas, endothelial Tie2-Cre-labeled fibroblasts were found in the IVS but not in the LVFW (Supplemental Figure 5A). Fibroblast lineage distribution in the RV following TAC remained similar to that of shamoperated controls, with the vast majority of fibroblasts within the $\mathrm{RV}$ free wall being derived from Wt1-Cre lineages, as found for the 
A

DAPI

Collagen1a1-GFP

Collagen Type I

Merge Trichrome (adjacent section)
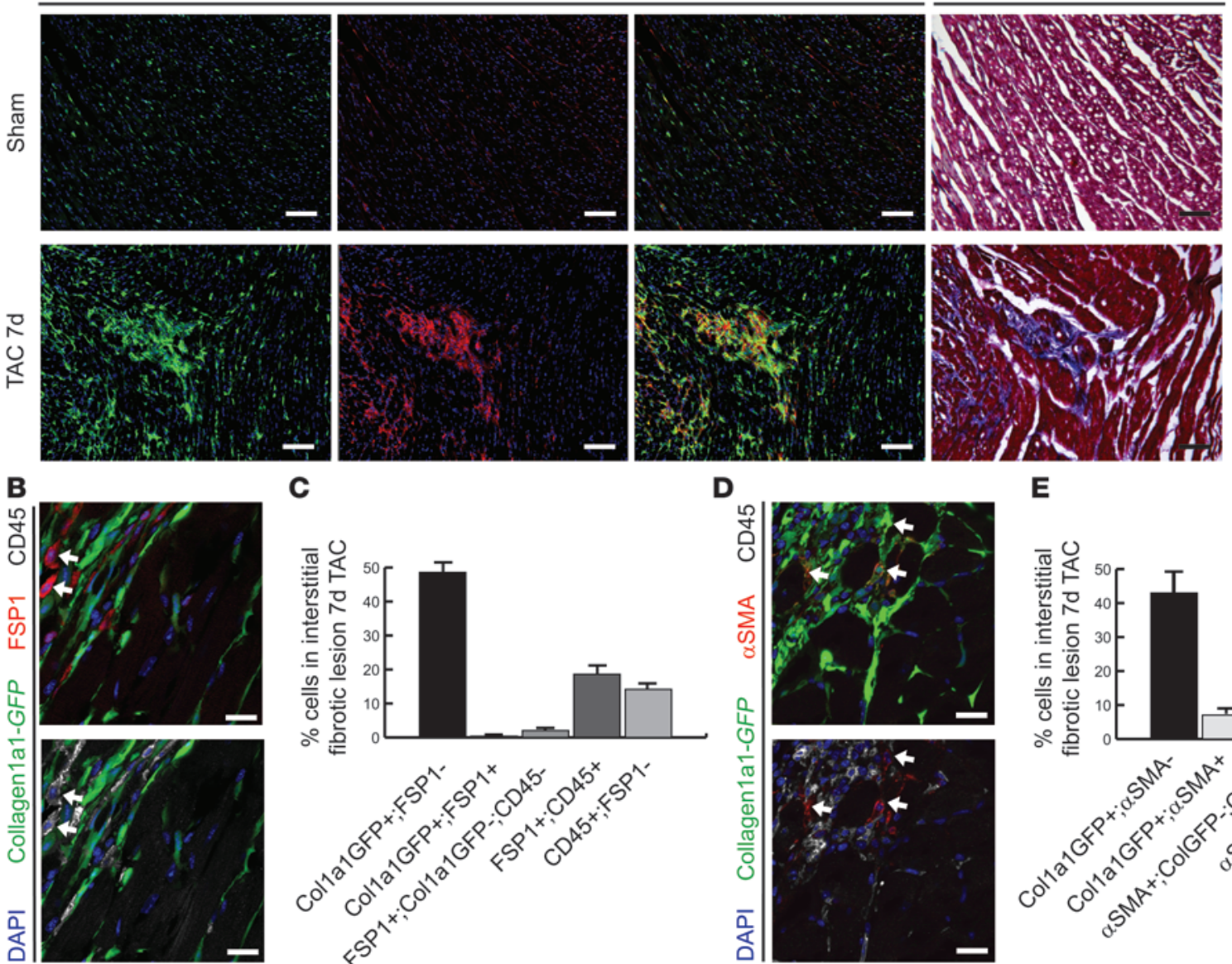

C

D

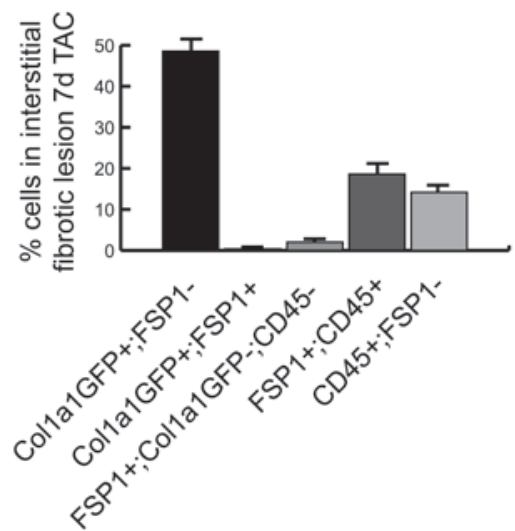

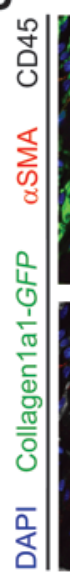

E
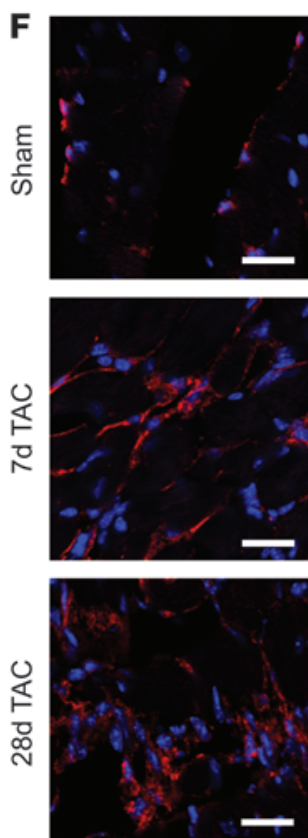

PDGFR $\alpha$

DAPI
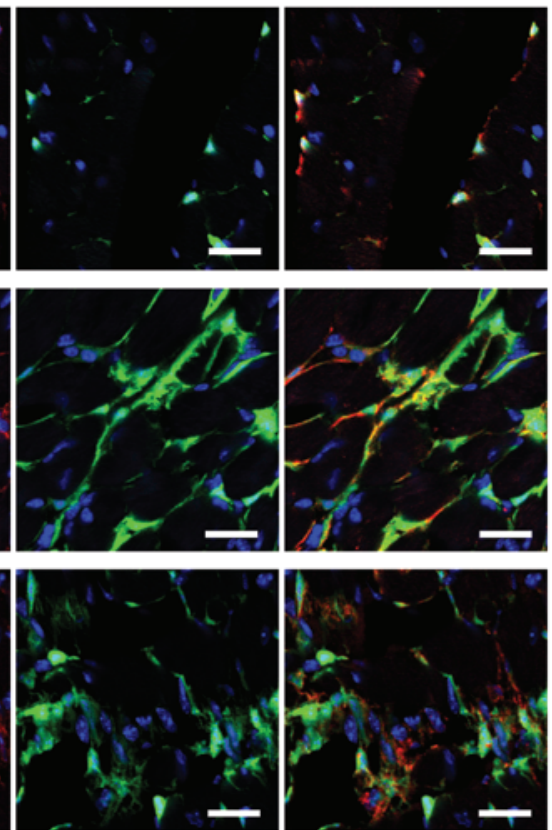

Collagen1a1-GFP

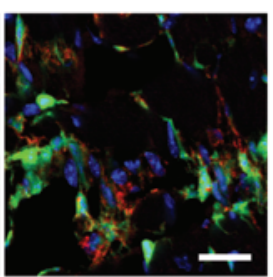

Merge

\section{Figure 2}

Fibroblast markers in pressure overload associated with fibrosis. (A) Collagen1a1-GFP+ fibroblast accumulation was associated with collagen type I deposition, as evidenced by immunofluorescence staining against collagen type I and trichrome staining in adjacent sections. Similar results were observed following 28 days of TAC. (B) Double-positive $\mathrm{CD} 45^{+} \mathrm{FSP} 1^{+}$cells within interstitial pathologic fibrotic lesions (arrows). The pathologic lesion is evident as an area rich in collagen-GFP+ cells and CD45+ leukocytes on the left of the images. No double-positive collagen1a1-GFP ${ }^{+}$CD $45^{+}$cells were observed. (C) Percentages of the total number of cells in interstitial fibrotic areas expressing collagen1a1-GFP, FSP1, and CD45 following 7 days of TAC. (D) $\alpha S M A$ expression in some collagen1a1-GFP+ fibroblasts (arrows) following 7 days of TAC. (E) Percentages of the total number of cells in interstitial fibrotic areas expressing collagen1a1-GFP, $\alpha$ SMA, and CD45 following 7 days of TAC. CD45+ cells were never collagen1a1-GFP+ (F) Overlap of collagen1a1-GFP and PDGFR $\alpha$ signals in sham and following 7 and 28 days of TAC. Histograms represent mean \pm SD of 12 fields from 3 mice. Scale bars: $100 \mu \mathrm{m}(\mathbf{A}) ; 20 \mu \mathrm{m}(\mathbf{B}, \mathbf{D}$, and F). 
A

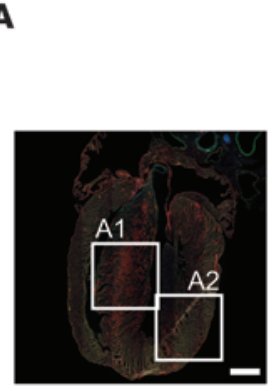

Wt1-cre:Rosa-tdT

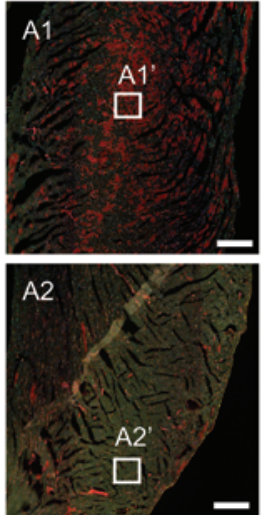

DAPI

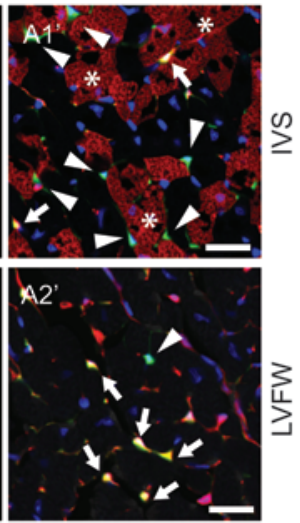

Collagen1a1-GFP
B

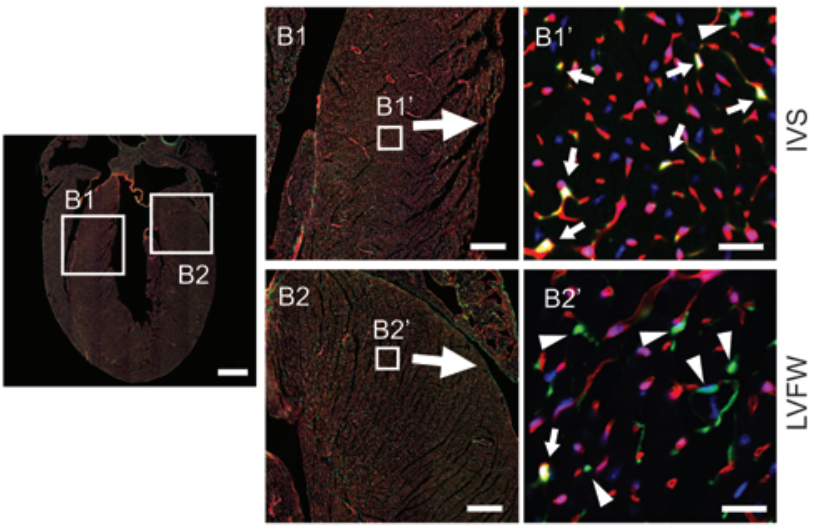

Tie2-cre;Rosa-tdT

DAPI

Collagen1a1-GFP
C

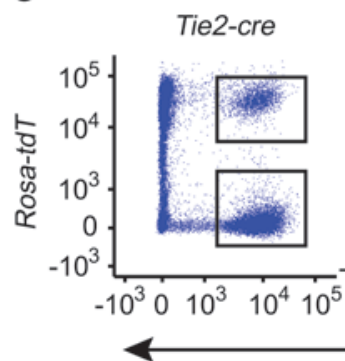

Wt1-cre

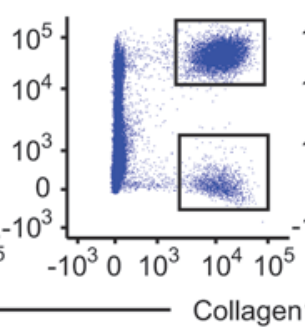

Wt1-cre+ Tie2-cre

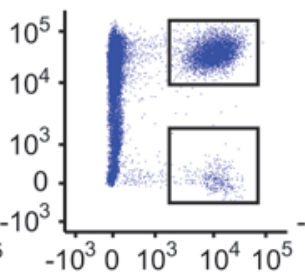

Vav-cre

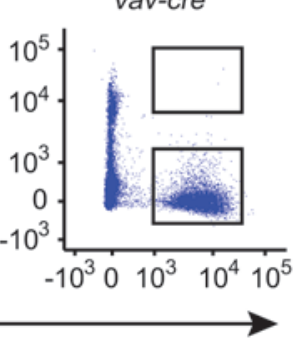

D

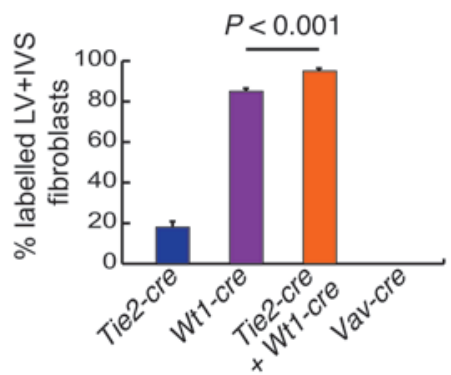

Figure 3

Complementary distribution patterns of Wt1-Cre and Tie2-Cre CF lineages. (A) Representative 4-chamber view of adult Wt1-Cre+/- collagen1a1-GFP+/- Rosa-tdT $T^{+/}$heart. IVS $\left(\mathrm{A} 1, \mathrm{~A} 1^{\prime}\right)$ contained few lineage-traced fibroblasts, whereas fibroblasts in the LVFW (A2, A2') were predominantly lineage traced (lineage traced, arrows; nonlineage traced, arrowheads). Labeled myocytes were abundant in the IVS (asterisks). (B) Representative Tie2-Cre ${ }^{+-}$collagen1a1-GFP+- Rosa-tdT $T^{+/}$heart showing Tie2-Cre lineage-traced fibroblasts were predominant in the IVS (B1, B1'), but most fibroblasts were nonlineage traced in the LVFW (B2, B2') (lineage traced, arrows; nonlineage traced, arrowheads). (C) Flow cytometry plots of dissociated LV and IVS from Tie2-Cre, Wt1-Cre, Wt1-Cre + Tie2-Cre, and Vav-Cre lineage-traced mice. Tie2-Cre and Wt1-Cre, but not Vav-Cre, labeled collagen1a1-GFP+ CF populations. (D) Quantification of the relative numbers of lineage-traced fibroblasts ( $n=3$ per group). Combining Wt1-Cre and Tie2-Cre resulted in labeling of approximately $95 \%$ of fibroblasts. Histograms represent average \pm SD. Data from Wt1-Cre and Wt1-Cre + Tie2-Cre groups were compared using unpaired Student's $t$ test. Scale bars: $1 \mathrm{~mm}(\mathbf{A}$ and B); $250 \mu \mathrm{m}$ (A1, A2, B1, B2); and $20 \mu \mathrm{m}\left(\mathrm{A} 1^{\prime}, \mathrm{A} 2^{\prime}, \mathrm{B} 1^{\prime}, \mathrm{B} 2^{\prime}\right)$.

LVFW (Supplemental Figure 5, C and D). Fibrosis was attenuated in the RV relative to that observed in the LV, as expected (24). Tie2 protein expression was observed in endothelium, but not in fibroblasts, both before and after TAC (Supplemental Figure 5E). Quantification of relative numbers of Tie2-Cre- and Wt1-Cre-labeled CFs in the LV and IVS by flow cytometry revealed that ratios of these lineages did not significantly change following pressure overload (Tie2-Cre- and Wt1-Cre-labeled CFs in sham-operated mice represented $19 \% \pm 1.5 \%$ and $86 \% \pm 0.4 \%$ of CFs, respectively; Figure 4 , $\mathrm{B}$ and $\mathrm{C})$. Tie2-Cre-labeled cells consisted of distinct populations of collagen1a1-GFP ${ }^{+}$fibroblasts, PECAM1 ${ }^{+}$endothelial cells, and $\mathrm{CD}_{4} 5^{+}$leukocytes (Supplemental Figure 6, A-C). Importantly, in double Tie2-Cre ${ }^{+/-} \mathrm{Wt1}$-Cre ${ }^{+/-}$collagen1a1-GFP ${ }^{+/-} \mathrm{Rosa}_{-}-\mathrm{td} \mathrm{T}^{+/-}$mice, approximately $94 \%$ of fibroblasts were lineage traced following 1 month of TAC, ruling out a significant contribution from other lineages (Figure 4C and Supplemental Figure 6D).

Hence, during hypertrophy, the distribution and relative proportions of activated Tie2-Cre and Wt1-Cre CF lineages were similar to those observed in healthy myocardium, consistent with accumulation of resident fibroblasts by proliferation. In keeping with this, analysis of fibroblasts from Tie2-Cre ${ }^{+/-}$collagen 1a1- $\mathrm{GFP}^{+/-}$ Rosa-td $T^{+/-}$mice following TAC demonstrated increased proliferation rates for both Tie2-Cre lineage-traced and nonlineage-traced collagen 1a1-GFP+ fibroblasts, with rates being similar in both populations, as shown by EdU labeling 4, 7, and 28 days after TAC (Figure 4D and Supplemental Figure 6E). Interestingly, proliferation was evident during the first week following surgery but had dropped markedly by 28 days. Apoptotic cleaved caspase- $3^{+}$cells were rare in the myocardium following TAC and were negative for collagen1a1-GFP (Supplemental Figure 6F). Although the foregoing data suggested that fibrotic fibroblasts were derived from resident Wt1-Cre or Tie2-Cre fibroblast lineages, it was important to further investigate the potential contribution of a number of other potential cell sources for pathological fibroblasts, including conversion of circulating hematopoietic progenitors, pathological EndoMT, and epicardial EMT.

Circulating hematopoietic progenitors do not give rise to fibroblasts following aortic banding. Vav-Cre recombination occurred specifically in hematopoietic cells at high rates; Vav-Cre labeled over $95 \%$ of $\mathrm{CD}^{4} 5^{+}$immune cells in hearts (Supplemental Figure 7A). 
A
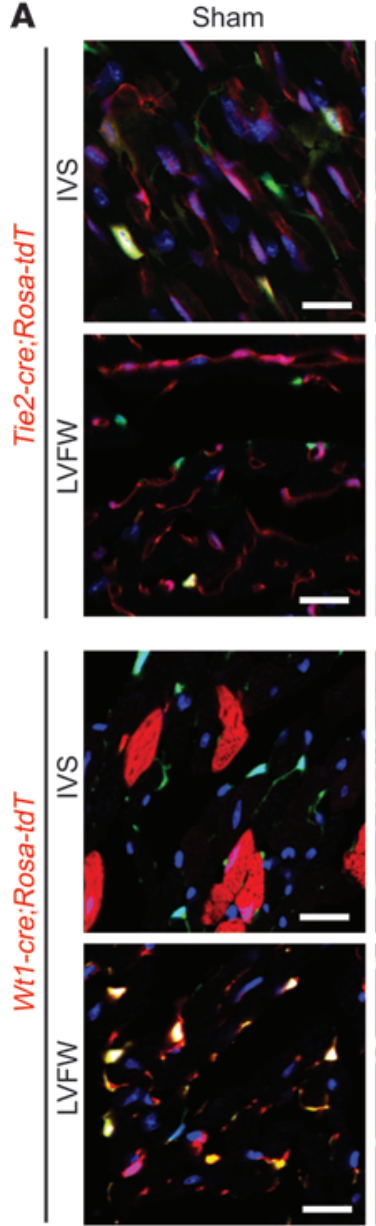

TAC 7d
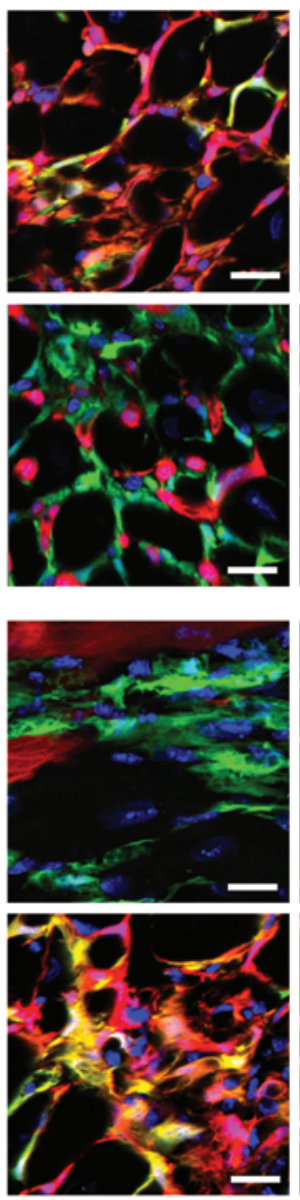

TAC 28d
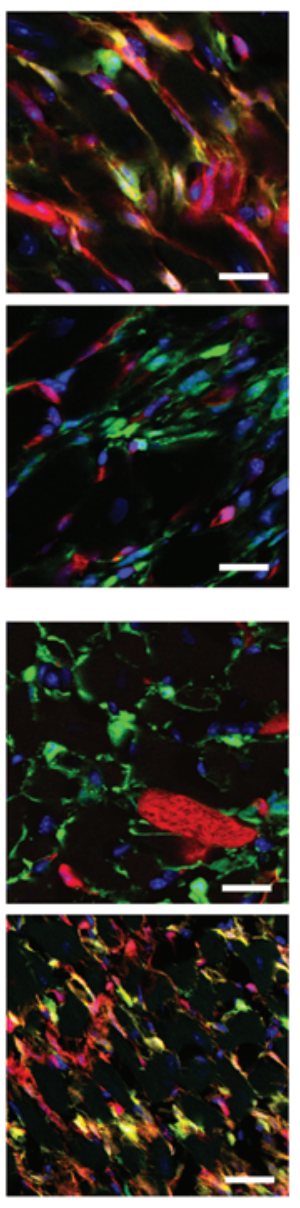

DAP

Collagen1a1-GFP

B

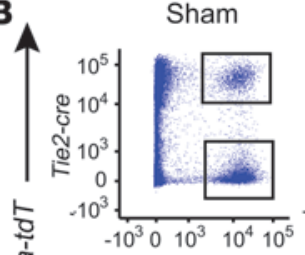

TAC 7d

TAC 28d
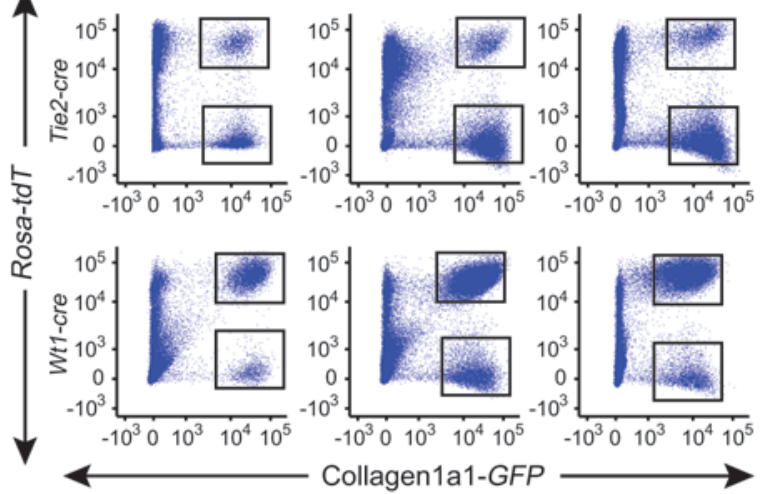

\section{Figure 4}

Fibroblasts accumulate by proliferation of resident lineages. (A) Confocal images showing Wt1-Cre and Tie2-Cre lineage-traced cells in shamoperated mice (7 days) and following 7 and 28 days pressure overload. The differential distribution of Wt1-Cre and Tie2-Cre fibroblast lineages in the IVS and LVFW was similar in sham-operated and fibrotic hearts. (B) Flow cytometry analysis of LV and IVS showing Wt1-Cre and Tie2-Cre lineage-traced collagen1a1-GFP+ fibroblasts in sham-operated animals (7 days) and following pressure overload (7 and 28 days). (C) Quantitative analysis of FACS data from Wt1-Cre and Tie2-Cre mice demonstrated that the relative numbers of lineage-traced fibroblasts did not significantly vary between sham- and TAC-operated animals, except for a small decrease in Wt1-Cre lineage-traced cells at 7 days of TAC. In double Wt1-Cre ${ }^{+/-}$ Tie2-Cre ${ }^{+/-}$collagen1a1-GFP+/- Rosa-tdT $T^{+/-}$mice, $94 \% \pm 0.8 \%$ of all fibroblasts were labeled following 28 days of TAC. S, sham-operated mice. (D) Quantification of proliferation rates of Tie2-Cre lineage-traced fibroblasts in the IVS and Tie2-Cre nonlineage-traced fibroblasts in the LVFW 4,7 and 28 days after surgery (trans-stenotic systolic pressure gradient [PG] day $4,76.7 \pm 8.2 \mathrm{mmHg}, n=3$ ). Cells were counted in $n=3$ mice per group, 6 fields per heart. NS, not significant $(P \geq 0.05) . P=0.01,{ }^{*} P<0.001$, compared with other groups, determined by ANOVA followed by Bonferroni's test. Data are shown as mean \pm SD. Scale bars: $20 \mu \mathrm{m}$.

Confocal and FACS analysis showed no evidence for Vav-Cre-labeled blood lineage-derived fibroblasts in hearts subjected to pressure overload (Figure 5). Almost all Vav-Cre Rosa-td $T^{+}$cells expressed the immune cell marker CD45 before and following TAC, except small subsets that were PECAM1+ (Supplemental Figure 7B).

Lineage tracing of adult endothelium shows no evidence for EndoMT following aortic banding. To further investigate pathological EndoMT of coronary vasculature in response to TAC (6), we generated VE-cadherin-CreERT2 ${ }^{+/-}$collagen 1a1-GFP $P^{+/-}$Rosa-td $T^{+/-}$mice and performed tamoxifen inductions in 5-week-old adults at baseline prior to TAC (Supplemental Figure 8, A and B). Following aortic banding, immunofluorescence analysis performed at either day 7 or day 28 after TAC in regions with high recombination demon- strated that VE-cadherin-CreERT2 Rosa-tdT labeled PECAM1 ${ }^{+}$ endothelium but not collagen 1a1-GFP+ fibroblasts (Figure $6 \mathrm{~A}$ and Supplemental Figure 5B). These observations were corroborated by flow cytometry analysis (Figure 6B). Hence, these data strongly suggested that adult endothelium does not give rise to CFs during pressure overload-induced fibrosis.

Lineage tracing of adult epicardium shows no evidence of epicardial EMT following aortic banding. Lineage tracing showed that myocardial infarction can activate epicardium, generating fibroblasts (9). To investigate whether epicardium also gives rise to fibroblasts in the setting of pressure overload, we used an inducible Wt1-CreERT2 in a Rosa-tdT indicator background. Tamoxifen inductions in 5 -week-old adults ( 3 consecutive days) lead to labeling of epicar- 
A

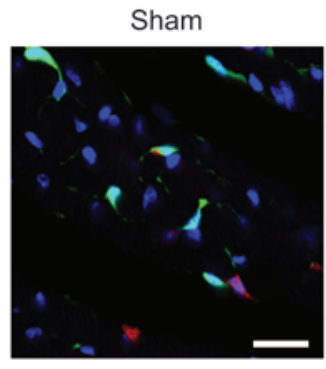

Vav-cre,Rosa-tdT

B

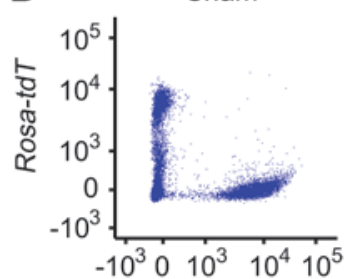

TAC 7d

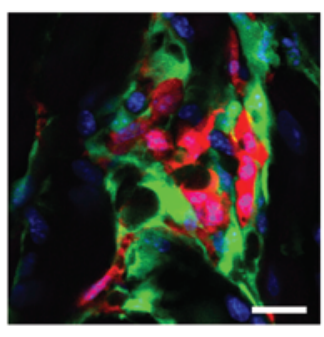

Collagen1a1-GFP

7d TAC

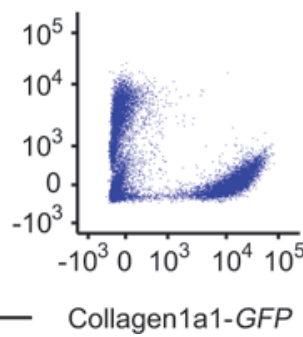

TAC 28d

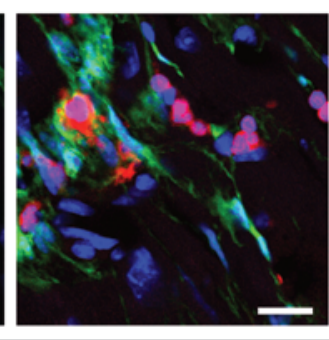

DAPI

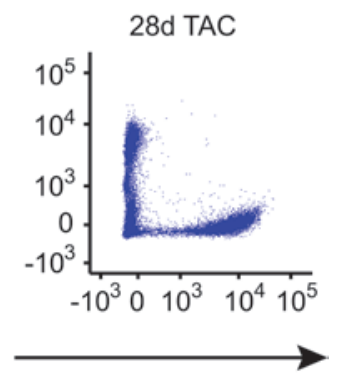

Figure 5

CFs do not derive from the hematopoietic lineage following pressure overload. (A) Confocal images showing a lack of collagen1a1-GFP+Vav-Cre lineage-traced cells in sham-operated hearts and following 7 and 28 days of TAC. (B) Flow cytometry plots showing that Vav-Cre lineage-traced cells were collagen1a1-GFP-. Images/plots are representative of at least 3 individuals. Scale bars: $20 \mu \mathrm{m}$.

dium, as previously described (9), and rare cells in the myocardium, which were negative for collagen1a1-GFP (Figure 7A). Epicardium expresses high levels of collagen type I (25) and was collagen-GFP+ at baseline and following surgery, as evidenced by colocalization of Wt1-CreERT2 Rosa-tdT and collagen1a1-GFP signals (Figure 7A, insets). We were unable to detect $\mathrm{Wt} 1$ protein expression in adult hearts by immunofluorescence, suggesting low levels of expression and underlining the sensitivity of the inducible reporter.

Following pressure overload, we did not observe any Wt1CreERT2 lineage-traced fibroblasts (Figure 7, A and B), suggesting that fibroblasts were not derived from epicardium. These results were further supported by similar experiments using a Tbx18-CreERT2 in a Rosa-tdT indicator background. Tamoxifen inductions in adult mice led to Rosa-tdT labeling of epicardium and $\mathrm{CD}_{146^{+}}$mural cells but not PDGFR $\alpha^{+}$fibroblasts (Supplemental Figure 9).

Fibroblasts of endothelial origin likely derive from endocardium during embryonic development. To gain further insight into embryonic origins of Tie2-Cre-labeled CFs, we investigated fibroblast origins at embryonic stages. Endothelium of the heart develops from two distinct sources: the sinus venosus and the endocardium $(26,27)$. A majority of mesenchymal cells within the AV valves is derived from EndoMT of a subset of endocardial cells, making this an attractive candidate for the source for the endothelially derived fibroblasts in myocardium $(22,28)$. Tie2-Cre lineage tracing lead to labeling of all endothelium/endocardium as well as collagen1a1-GFP fibroblasts that were first seen within cardiac tissue adjacent to $\mathrm{AV}$ canal cushions at E12.5 (Figure 8A). With time, these cells were localized progressively further from the cushions, in partic-

ular within the septum, consistent with their being derived from EndoMT of cushion endocardium and migrating out from this region. The collagen1a1-GFP signal was restricted to PDGFR $\alpha^{+}$fibroblasts and not PECAM1 ${ }^{+}$endothelium in embryonic myocardium (Figure 8C) but also labeled the adventitia of large vessels outside the heart (aorta, pulmonary vein) and epicardium (Figure 8A). PDGFR $\alpha$ was not expressed by vascular smooth muscle during development or in adult heart (Supplemental Figure 10, A and B).

The recently described Nfatc1-Cre (27) labels both endocardium and coronary endothelium at stages when fibroblasts are entering the myocardium (Supplemental Figure 10C), thus we could not selectively attribute labeled fibroblasts to an endocardial origin using this Cre driver. However, Nfatc1-Cre revealed a similar distribution of lineage-traced fibroblasts to that observed using Tie2-Cre, confirming the endothelial origin of a subpopulation of fibroblasts (Supplemental Figure 10D).

To further investigate a potential endocardial origin of endothelially derived fibroblasts, we performed pulse labeling of endothelium, including endocardium, in embryos using inducible VE-cadherinCreERT2, adding tamoxifen at times before (E7.5E8.5) or after (E9.5-E10.5) the onset of endocardial EndoMT (Figure 8B). Following early induction with tamoxifen, we observed Rosa-tdT lineage labeling of collagen 1a1-GFP+ valve mesenchyme, a marked number of CFs throughout the septum, and, to a lesser extent, CFs in the ventricular free walls at E17.5 (Figure 8C). However, inductions of VE-cadherin-CreERT2 at E9.5 or later did not result in labeling of fibroblasts in valves or within cardiac tissue, despite similar levels of endocardial/endothelial labeling with either induction (Figure 8D and Supplemental Figure 11, A and B). Consistent with a previous report (26), early induction led to Cre-mediated Rosa-tdT expression specifically in VE-cadherin ${ }^{+}$endothelium/endocardium and AV cushions but not in $\mathrm{Wt}^{+} 1^{+}$proepicardial or $\mathrm{Wt} 1^{+}$epicardial cells (Supplemental Figure 11, C and D). These results, along with the distinct spatial distribution of CF lineages observed with constitutive Tie2-Cre or Nfatc1-Cre and Wt1-Cre or Tbx18-Cre, strongly suggested that mesenchymal cells produced by EndoMT of the endocardium during cushion development contributed to CFs within myocardium.

Epicardial and endothelial/endocardial resident fibroblast lineages have similar fibrotic responses following aortic banding. Finally, to investigate whether the two developmentally distinct CF populations exhibited distinct expression profiles in response to cardiac injury/stress and therefore might necessitate distinct therapeutic targeting, we performed microarray analysis on FACS-sorted fibroblast lineages (see Methods). Tie2-Cre lineage-traced CFs (Tie2-Cre Rosa-td $T^{4}$, collagen1a1-GFP $P^{+}$, non-Tie2-Cre lineage-traced CFs (Tie2-Cre Rosa$t d T^{-}$, collagen 1a1-GFP $P^{+}$, and endothelial cells (PECAM1 ${ }^{+}$) were sorted from dissociated LV and IVS of sham-operated and banded hearts at the onset of fibrosis, 1 week after surgery. A cross-comparison matrix (Figure 9A) clearly distinguished both fibroblast populations from PECAM1 ${ }^{+}$endothelial cells. Tie2-Cre Rosa-td $T^{+}$ and Tie2-Cre Rosa-tdT- fibroblast groups were closely correlated in either sham-operated or TAC groups, suggesting they had very similar changes in gene expression in response to TAC. Array data 
A
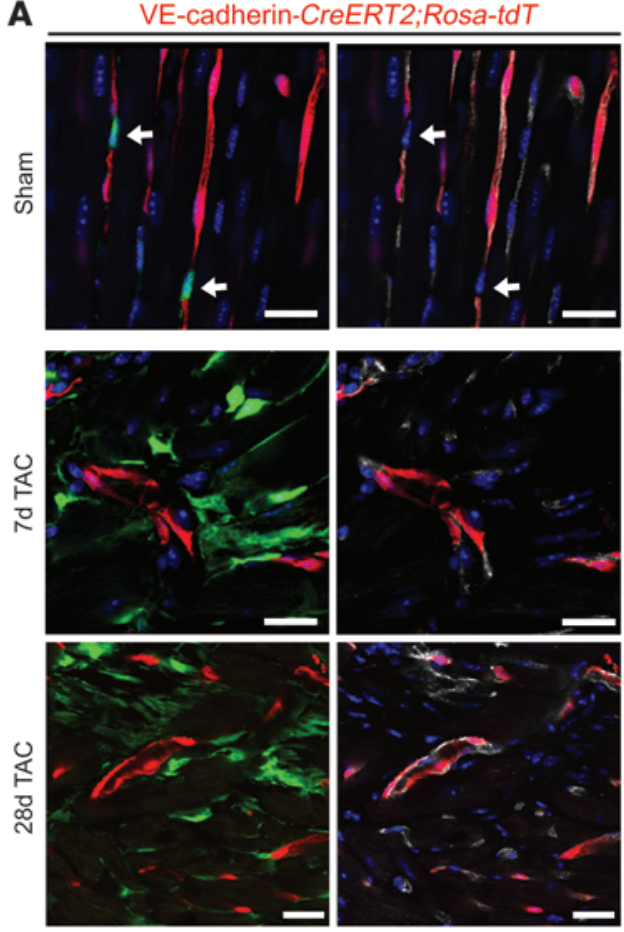

DAPI

Collagen1a1-GFP PECAM1
B
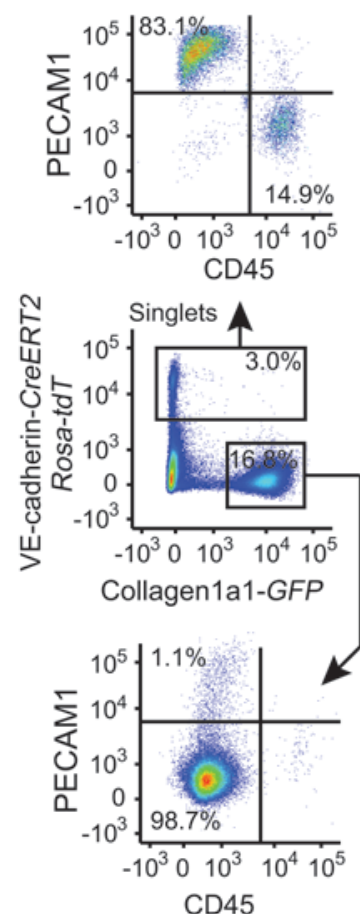

$7 d$ TAC
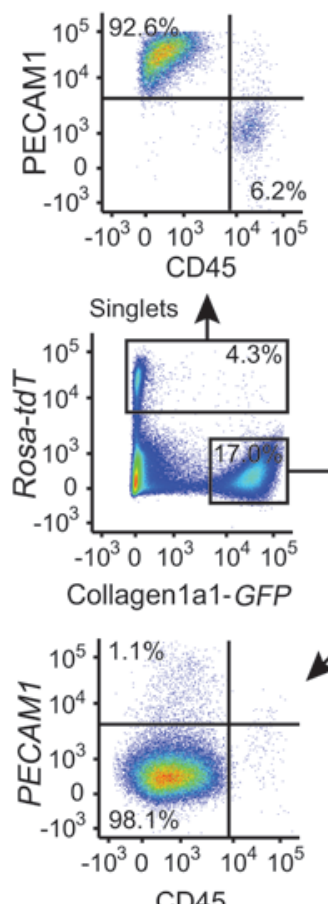

28d TAC
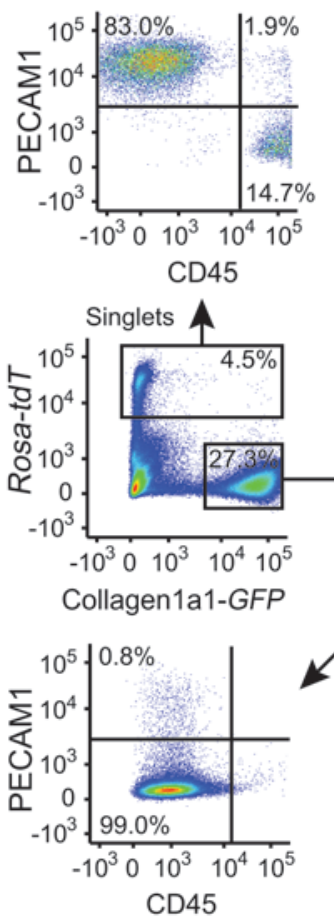

Figure 6

Lineage tracing of adult cardiac endothelium following TAC. (A) Confocal analysis of LV and IVS in sham-operated mice or following 7 and 28 days of pressure overload. Lineage-traced cells (red) were PECAM1+ endothelial cells (gray) and were never collagen1a1-GFP+ fibroblasts (green) (representative of 3 mice per group). (B) Flow cytometry analysis of dissociated LV and IVS from VE-cadherin-CreERT2+/- collagen1a1-GFP+/Rosa-td $T^{+/}$lineage-traced sham-operated hearts and following 7 and 28 days of TAC. Plots are representative of 2 to 3 individuals. Cell populations are identified with Rosa-tdT (VE-cadherin-CreERT2 lineage traced), collagen1a1-GFP (fibroblasts), PECAM1 (endothelium), and CD45 (leukocytes). In fibrotic hearts as well as in sham-operated hearts, VE-cadherin-CreERT2 lineage-traced cells (Rosa-tdT+, top gates) were PECAM1+ or $\mathrm{CD}_{4} 5^{+}$, indicating that they had not adopted a fibroblast fate. Accordingly, VE-cadherin-CreERT2 Rosa-tdT+ cells were collagen1a1-GFPThe collagen1a1-GFP+ fibroblasts were PECAM- and CD45- (bottom gates). In all mice, a small percentage of collagen1a1-GFP+ $(\sim 1 \%)$ were PECAM1+, due to collagen expression in some endocardial cells. Scale bars: $20 \mu \mathrm{m}$.

from these 6 groups were then clustered based on differentially expressed genes. We could clearly distinguish CF, endothelial, and TAC-responsive clusters of transcripts (Figure 9B). Array data were validated by quantitative RT-PCR (qRT-PCR) of selected genes from cluster 1 (TAC response in CFs) (Wisp1, Col11a1), cluster 2 (CF-specific genes) (Col1a1, Ddr2, Pdgfra, Postn), and cluster 3 (endothelial-specific genes) (Pecam1, Cdh5) in independent samples (Supplemental Figure 12A). GO term analysis was performed on the main clusters, revealing a very significant number of genes associated with cell cycle to be upregulated in fibroblasts after TAC (Supplemental Figure 12B). Interestingly, among other gene groups specifically upregulated in fibroblasts following aortic banding, we identified extracellular matrix and negative regulation of cell death (Supplemental Figure 11, B and C, and Supplemental Excel File 1).

\section{Discussion}

Our studies have revealed a novel paradigm for the origins of CFs in pathological fibrosis (Figure 9C). We performed lineage-tracing experiments relying on robust markers for CFs, the collagen1a1-GFP reporter line, and PDGFR $\alpha$, specifically coexpressed by fibroblasts. We found that, following pressure overload, fibro- blast accumulation resulted from proliferation of two resident lineages: a previously described lineage of epicardial origin and a novel lineage derived from endocardium/endothelium. These lineages responded similarly to pressure overload, suggesting they may be amenable to similar therapeutic targeting.

Recently, EndoMT promoted by cardiac injury, including pressure overload, has been reported to make a substantial contribution to fibroblast accumulation in response to injury $(6,7)$. Hence, targeting EndoMT is currently considered a powerful therapeutic approach, enabling the preservation of the vasculature while reducing fibroblast numbers in failing hearts. However, only one study has reported EndoMT in adult cardiac fibrosis based on lineage tracing (6). This study, which reports that Tie1-Cre lineage-traced FSP $1^{+}$cells present following pressure overload were fibroblasts generated by pathologic EndoMT, has major limitations. We found FSP1 to be more readily expressed by $\mathrm{CD}_{4} 5^{+}$immune cells than fibroblasts. Moreover, Tie1-Cre labels immune cells (29), hence the presence of Tie1-Cre lineage-traced $\mathrm{FSP}^{+}$immune cells can be expected in the absence of EndoMT, particularly in contexts involving inflammation. Finally, the presence of a developmentally derived fibroblast population of endothelial origin had not been excluded. 

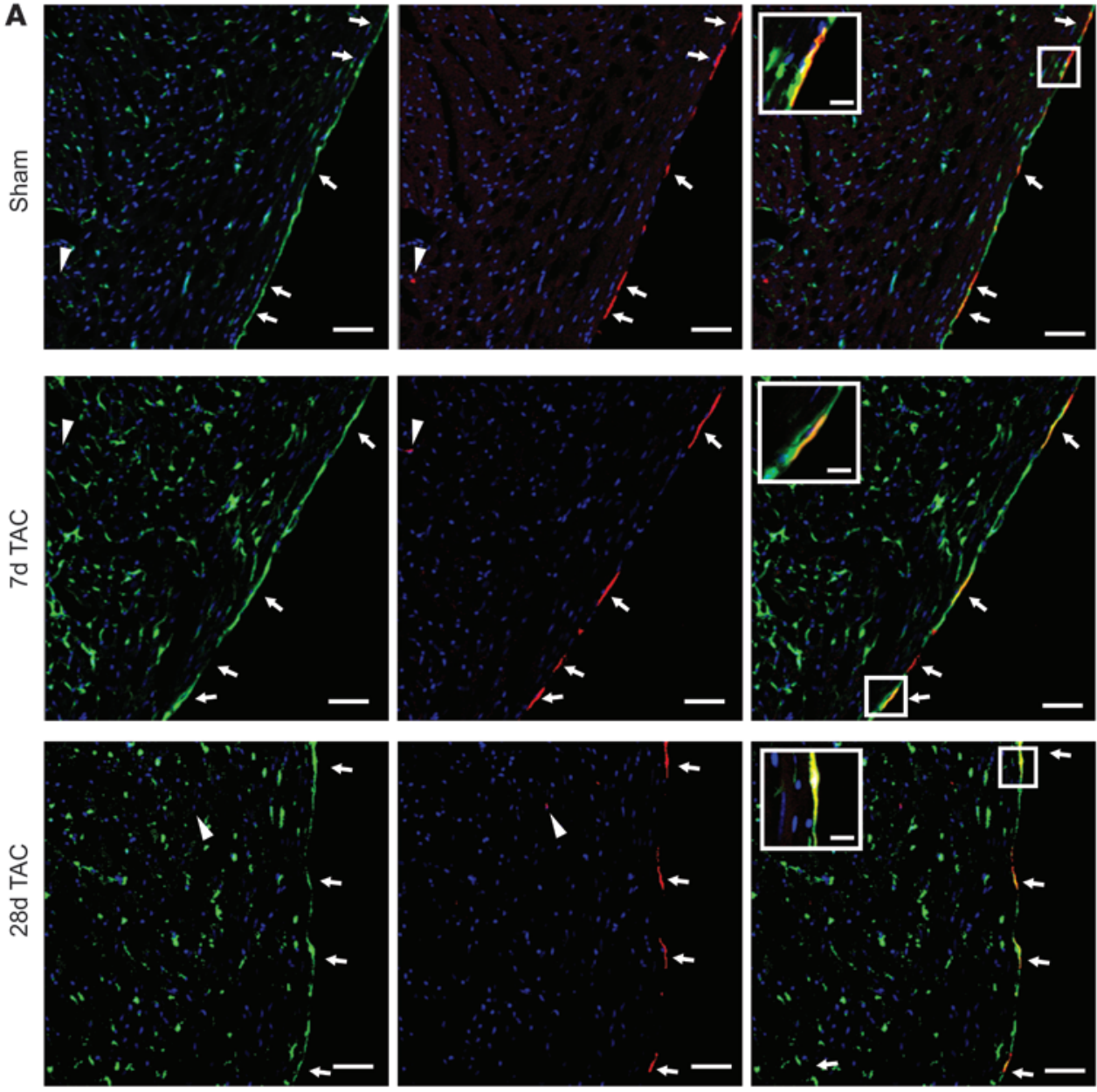

Collagen1a1-GFP

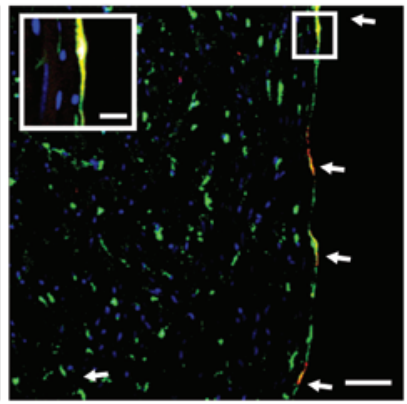

Wt1-CreERT2;Rosa-tdT

\section{Figure 7}

Epicardium does not give rise to fibroblasts following pressure overload. (A) Confocal images showing labeling in epicardium (arrows) but not in collagen1a1-GFP+ fibroblasts of sham-operated Wt1-CreERT2 ${ }^{+/-}$ collagen1a1-GFP ${ }^{+/-}$Rosa-td $T^{+/-}$mice and following 7 and 28 days of pressure overload. Rare lineage-traced collagen1a1-GFP- cells (arrowheads) were present in the interstitium of sham-operated and hypertrophic hearts. Scale bars: $50 \mu \mathrm{m}$. (B) No collagen1a1-GFP+ fibroblasts were labeled in fibrotic areas following 7 days of TAC. Similar results were observed following 28 days of TAC. Scale bars: $20 \mu \mathrm{m} ; 5 \mu \mathrm{m}$ (insets).

B

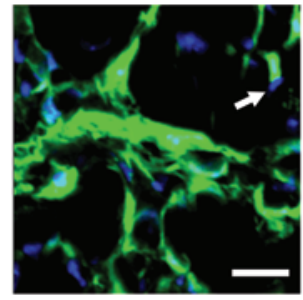

DAPI

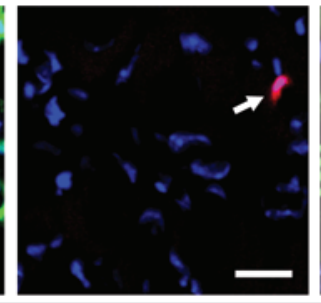

Collagen1a1-GFP

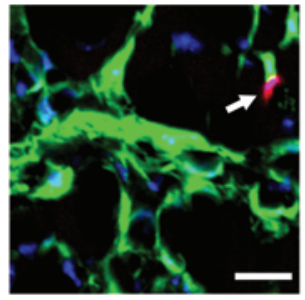

Wt1-CreERT2;Rosa-tdT
Because of the foregoing issues with FSP1 as a fibroblast marker, we set out to quantitatively assess potential fibroblast markers in both interstitial and perivascular fibrotic areas after TAC. Our observations on FSP1 expression within the heart were consistent with a growing body of evidence showing that FSP1 alone is an unreliable marker for fibroblasts in heart (11) and other organs $(10,30)$. We found that in fibrotic hearts only a small subset of perivascular fibroblasts expressed FSP1, while no interstitial fibroblasts were found to express FSP1. Interestingly, recent studies have demonstrated distinct gene expression in perivascular versus interstitial fibroblasts (31). Another marker, $\alpha$ SMA, is widely used to identify activated fibroblasts, otherwise known as myofibroblasts. In the context of pressure overload-induced fibrosis, we found that only approximately $15 \%$ of fibroblasts in interstitial fibrotic regions and none in perivascular fibrotic regions expressed $\alpha$ SMA. This shows that $\alpha$ SMA expression does not comprehensively label fibroblasts responsible for fibrosis. aSMA expression has been associated with contractility rather than extracellular matrix production (32). Furthermore, aSMA is also highly expressed in smooth muscle cells and pericytes. These observations underscore potential limitations of using FSP1 or $\alpha$ SMA alone to mark fibrotic fibroblasts, at least in pressure overload models. In contrast, the collagen1a1-GFP transgene (12) and PDGFR $\alpha$ (14) comprehensively and selectively marked fibroblasts both in normal heart and throughout regions of both interstitial and perivascular fibrosis. We therefore used these markers to reexamine the origins of CFs both during development and in response to pressure overload. 
A

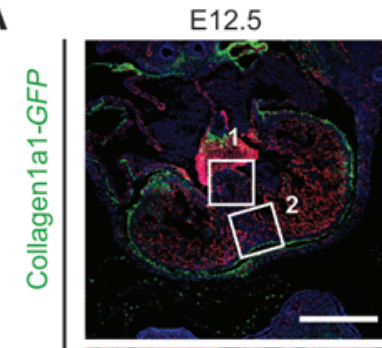

$\overline{\frac{1}{a}}$
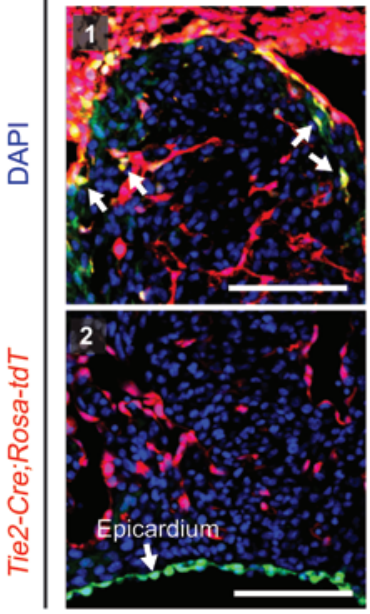

E15.5
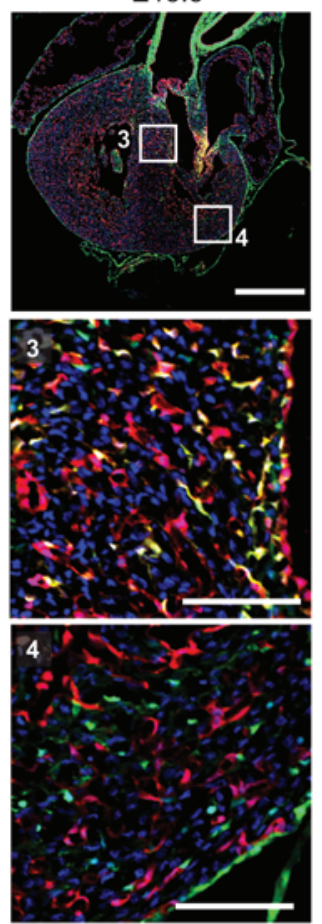

E18.5
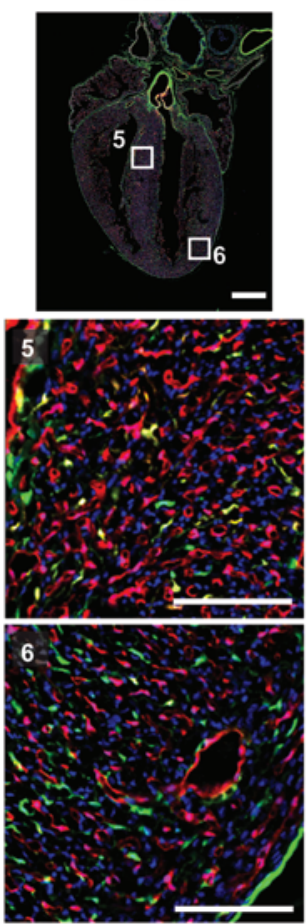

P7
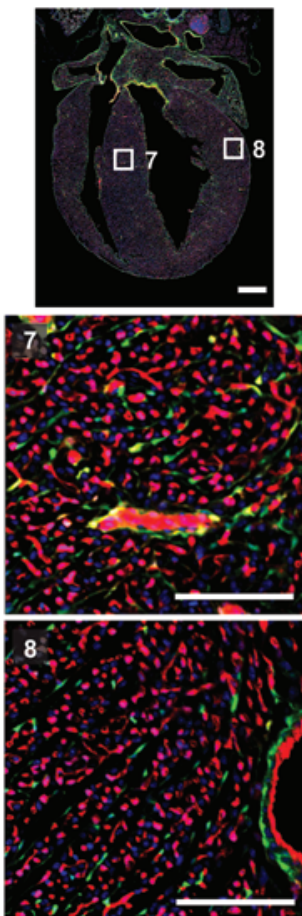

B

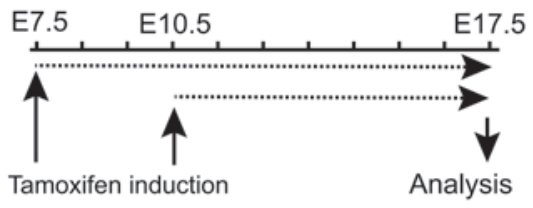

D

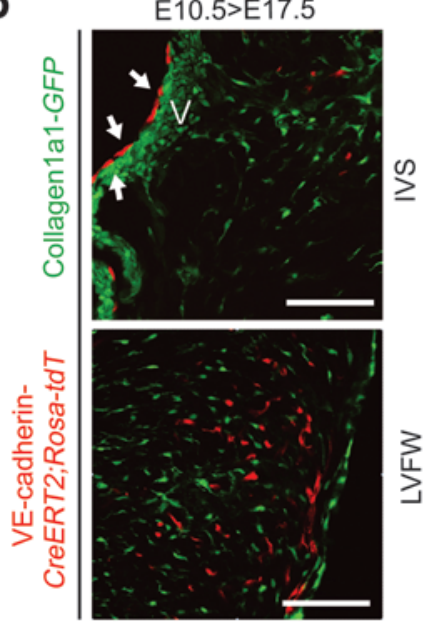

C

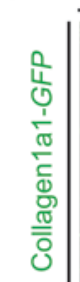

E7.5>E17.5
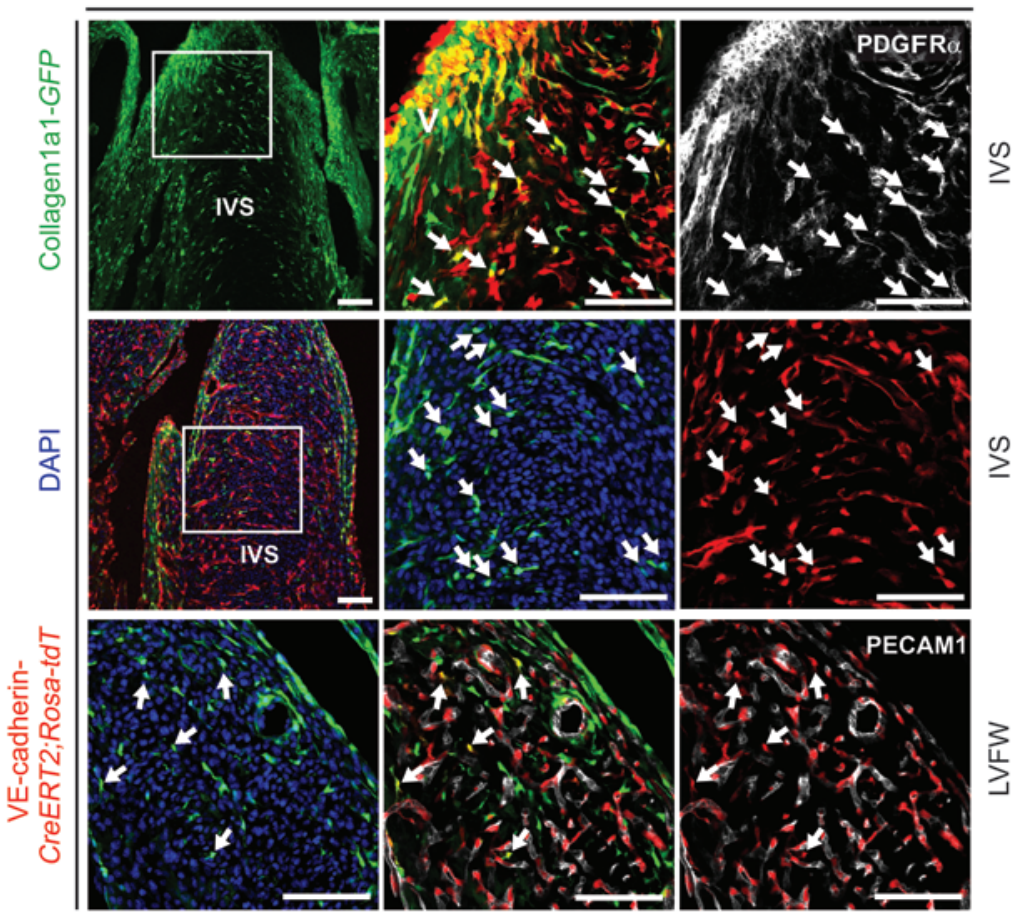

Figure 8

Analysis of the fate Tie2-Cre and of VE-cadherin-CreERT2 lineage-traced cells during early development. (A) Time course showing Tie2-Cre+/collagen1a1-GFP+/ Rosa-tdT+/- embryonic hearts at E12.5, E15.5, E18.5, and P7. Tie2-Cre lineage-traced fibroblasts were first visible within the myocardium close to the AV canal cushion at E12.5 (arrows, image A1) and subsequently populated the IVS. (B) Timing of tamoxifen inductions for VE-cadherin-CreERT2 embryos. (C) Inductions at E7.5 resulted in labeling of collagen1a1-GFP+/PDGFRo ${ }^{+}$valve mesenchyme (V) and interstitial fibroblasts (arrows) in the upper septum by E17.5. Many VE-cadherin-CreERT2 lineage-traced fibroblasts were also found migrating into the lower septum (arrows). Far less abundant VE-cadherin-CreERT2 lineage-traced PECAM1- fibroblasts were found in the ventricular free walls (arrows). (D) Induction at E10.5 resulted in labeling of endocardium (arrows) but not valve mesenchyme or interstitial CFs. Scale bars: $500 \mu \mathrm{m}$ (A, 4-chamber images); $100 \mu \mathrm{m}$ (A, bottom 2 rows, C, and D). 
A

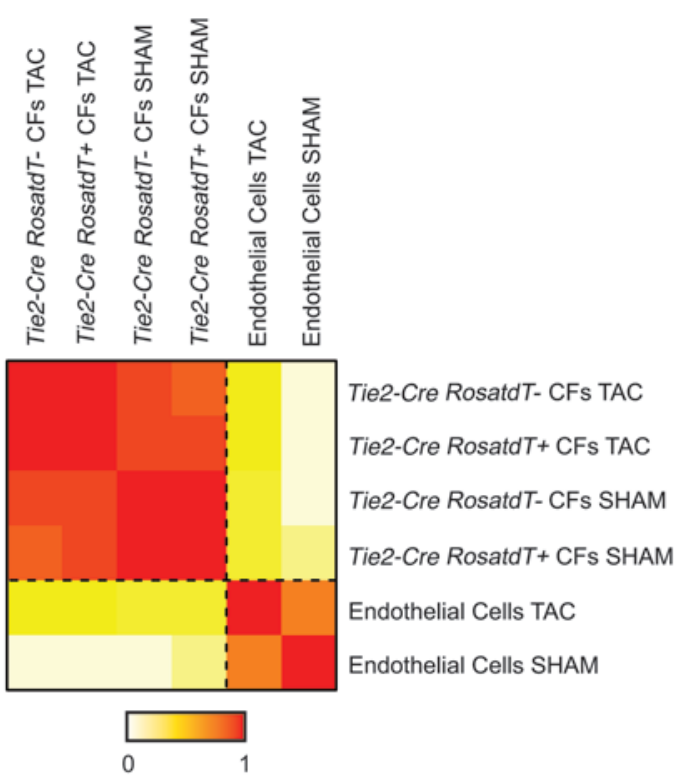

C

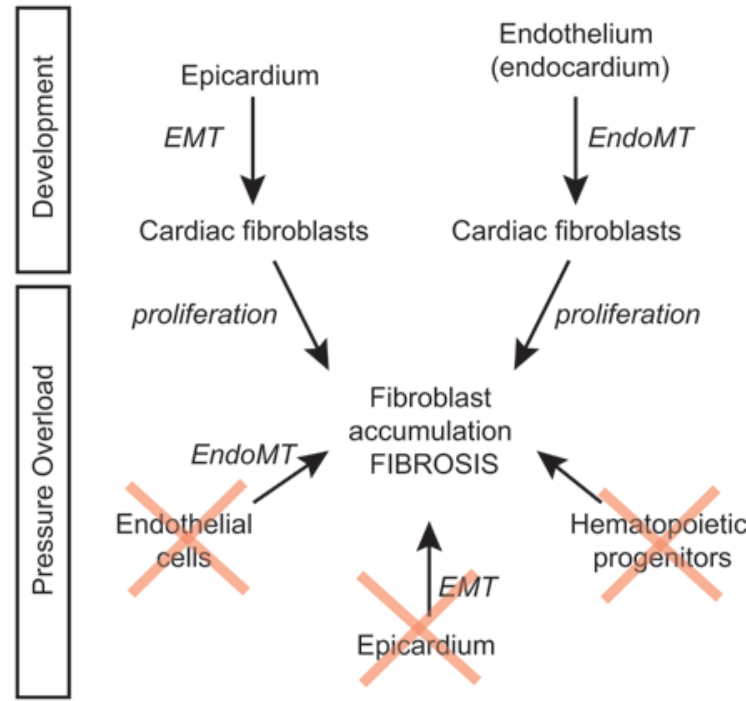

B
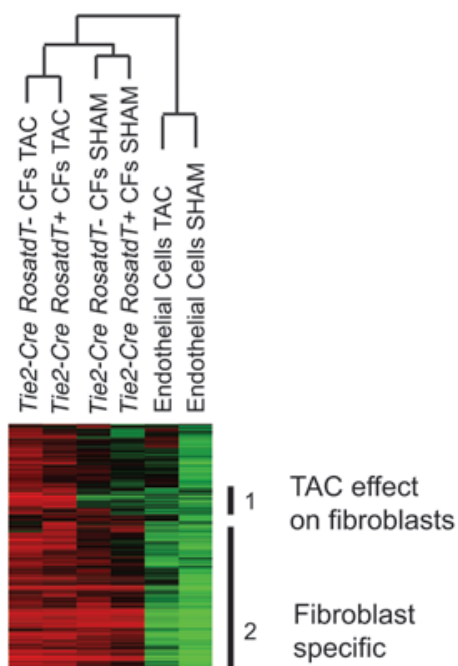

specific

Endothelial

cell specific

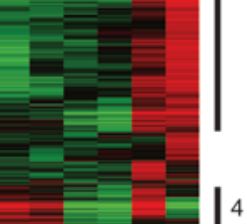

on fibroblasts +

endothelial cells

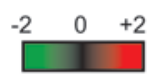

\section{Figure 9}

Gene profiling of fibroblast lineages and endothelium following pressure overload. (A) Heat plot representing all possible pairwise HOPACH correlation (euclidean) values. Darkest values indicate a high level of correlation ( $=1$ across the diagonal), and lighter color indicates lower correlation. A clear segregation of fibroblast and endothelial expression profiles is evident. (B) Hierarchical clustering of genes up or down 2-fold in at least one group revealed clusters of genes specific to fibroblasts following TAC (cluster 1), fibroblasts (cluster 2), endothelial cells (cluster 3 ), and fibroblasts and endothelial cells following pressure overload (cluster 4). (C) Proposed model for fibroblast origins based on findings from this study. Fibroblast accumulation during fibrosis resulting from pressure overload results from the proliferation of distinctly distributed resident epicardial- and endothelial-derived fibroblast populations. Fibrosis does not result from EndoMT, epicardial EMT, or recruitment of hematopoietic fibroblast progenitors. The similar genetic profiles and behavior of these fibroblast populations suggests that they could be targeted in a similar way to alleviate fibrosis.

Currently, it is presumed that all fibroblasts within normal myocardium are derived from epicardium, although this has not been directly assessed by fate mapping (13). The distribution pattern of Wt1-Cre-labeled epicardial CF lineages showed that epicardially derived CFs populated the myocardial free walls, as expected. However, surprisingly, it was evident that fibroblasts within the IVS were derived from another source. Endocardial EndoMT occurs proximal to this region and generates the majority of the mesenchyme in the $\mathrm{AV}$ valves $(22,28)$, making it a strong candidate. We confirmed an endothelial origin for these fibroblasts using constitutive Tie2-Cre, notably showing a complementary distribution of Tie2-Cre- and Wt1-Cre-labeled fibroblasts. Furthermore, quantitative flow cytometry analysis showed that the vast majority of CFs were labeled in double $\mathrm{Wt1}-\mathrm{Cre}^{+/-} \mathrm{Tie2}-\mathrm{Cre}^{+/-}$collagen1a1-GFP+/- 
Rosa-td $T^{4 /-}$ mice. Lack of labeling of a small population could have resulted from incomplete efficiency of the Cre drivers or a minor contribution from another lineage. Furthermore, limited overlap between the Cre expression domains could result from Wt1 expression in a small subset of endothelium (33). Importantly, this also suggested that fibroblasts derived from epicardial cells not labeled by Wt1-Cre, as recently reported (34), were infrequent.

Following pressure overload, we found that the relative distribution and proportion of endothelium- and epicardium-derived fibroblasts remained similar to those observed in sham-operated animals. This suggested that fibroblasts do not migrate to any significant extent following pressure overload. Each of these fibroblast populations also proliferated at similar rates following pressure overload, with proliferation peaking within the first week following surgery and returning to near-baseline levels at 28 days. Furthermore, in contrast to tissues with regenerative capacity, such as the liver, in which the resolution of fibrotic lesions is associated with fibroblasts undergoing apoptosis (35), we did not observe significant apoptosis of fibroblasts in heart. Together, these observations were consistent with accumulation of fibroblasts after TAC, resulting from acute proliferation of these two resident fibroblast populations.

Despite these observations, which suggested that resident fibroblasts were principal contributors to fibrosis with TAC, we also investigated potential alternate sources of fibrotic fibroblasts. Labeling of adult endothelium prior to TAC by tamoxifen induction of VEcadherin-CreERT2 $2^{+/-}$Rosa-td $T^{+/-}$mice did not result in the appearance of lineage-labeled fibroblasts following TAC. This observation suggested that EndoMT did not appreciably contribute to fibrotic fibroblast accumulation consequent to pressure overload.

Other potential sources of fibroblasts were circulating hematopoietic cells and reactivation of epicardial EMT. Although recruitment of hematopoietic progenitors has been reported previously for the hypertrophy model (6), our Vav-Cre genetic lineage did not show any contribution from blood. As previous studies used FSP1 to identify fibroblasts of hematopoietic origin, it is likely that most of these cells were in fact immune cells. Our results in the pressure overload model do not, however, rule out a contribution from blood-borne fibroblasts to fibrosis in other disease contexts, such as myocardial infarction $(8,36)$. Of note, we found a small subset of Vav-Cre lineage-traced endothelial cells. This could have resulted from a minor contribution of blood cells to endothelium, as previously suggested $(37,38)$, or low ectopic Vav-Cre expression in endothelial cells. Finally, although fibroblast progenitors are reportedly hematopoietic in nature (39) and our data show that a vast majority of fibroblasts (>94\%) were labeled in double Tie2-Cre Wt1-Cre-positive mice, we cannot rule out a small contribution of nonhematopoietic circulating fibroblast progenitors to fibrosis.

Epicardial EMT has long been identified as a source of fibroblasts during development, and, more recently, it has been identified as a source of fibroblasts in the myocardial infarction model (9). Histologically, we did not observe an activation/thickening of the epicardium in the TAC model, in agreement with a recent report comparing pressure overload and myocardial infarction models (40). Consistent with this, using inducible Wt1-CreERT2 and Tbx18-CreERT2 mouse lines that label epicardium but not fibroblasts, we did not identify a contribution of adult epicardium to fibroblasts following aortic banding. This latter result is also in agreement with our data indicating that the proportion of con- stitutive Wt1-Cre-labeled fibroblasts remained constant following pressure overload. In this context, it is important to note that, contrary to the myocardial infarction model described previously (9), fibrotic lesions were not intimately associated with epicardium in our pressure overload model. The distribution of epicardially and endothelially derived fibroblast lineages suggested that the latter may derive from endocardial EMT during development. Distributions of both Tie2-Cre and Nfatc1-Cre lineage-traced fibroblasts were in agreement with this possibility. As Nfatc1-Cre extensively labeled coronary endothelium, we were unable to definitively demonstrate that the labeled fibroblasts were from endocardium. However, we observed abundant VE-cadherin-CreERT2-labeled fibroblasts in septum of lineage-traced embryos induced with tamoxifen before, but not after, endocardial EMT. Together, these data suggested that most, if not all, Tie2-Cre-labeled fibroblasts within the IVS or LV derived from AV canal endocardium undergoing EMT during cushion formation.

Although activated CFs arose from two developmentally distinct populations, transcriptional profiling of FACS-sorted cells showed that both Tie2-Cre lineage-traced and nonlineage-traced fibroblasts responded in a similar manner to pressure overload and, therefore, may be amenable to joint therapeutic targeting. Notably, GO term analysis revealed genes associated with increased proliferation as being highly significantly upregulated. TGF- $\beta$-mediated proliferation of nonmyocytes has been reported to underlie fibrosis in a mouse model of hypertrophic cardiomyopathy (41). Such signaling, amplified in areas subjected to increased stress, could underlie the development of distinct fibrotic lesions, which we observed throughout the myocardium. However, we cannot rule out that a highly responsive subset of fibroblasts exists within the resident lineages, and this needs to be further investigated. Other groups of genes activated included the pathway "negative regulation of cell death," a result supported by a lack of cleaved caspase- $3^{+}$apoptotic fibroblasts in hypertrophic heart. Interestingly, it has been shown in the context of liver regeneration that resolution of the scar involves programmed cell death of activated extracellular matrix-producing hepatic stellate cells (35). These observations suggest that this group of genes may constitute a potentially important therapeutic target.

In summary, our studies have demonstrated that, following pressure overload, fibroblast accumulation results from the proliferation of two tissue-resident, developmentally distinct CF populations. However, fibroblasts were not derived from EndoMT, epicardial EMT, or hematopoietic cells, as previously reported.

\section{Methods}

Mice. Wt1-Cre (19), Tie2-Cre (42), Vav-Cre (43), VE-cadherin-CreERT2 (44), Tbx18-Cre (5), Wt1-CreERT2 (9), and Nfatc1-Cre (27) mice have been described previously. The transgenic collagen1a1-GFP reporter line (12) and Rosa-tdT-Cre reporter line (20) have been described previously.

To generate an inducible Cre line under the control of Tbx18 regulatory sequences (Tbx18-CreERT2) a cassette containing a Cre-ERT2 coding sequence, followed by a neomycin resistance gene flanked by FRT sites (FRT-mclNeo), was knocked into the first exon of the Tbx18 gene, disrupting expression of the endogenous gene. Cre-positive progeny were identified by PCR using generic CRE primers. All mice were on a Black Swiss background.

Tissue preparation for histology. Hearts were removed, perfused through the aorta with HBSS, fixed in 4\% PFA overnight, dehydrated in $25 \%$ sucrose, embedded in OCT/sucrose, and sectioned (10-40 $\mu \mathrm{m})$. 
Embryos were fixed in 4\% PFA overnight, dehydrated in 20\% sucrose, embedded in OCT/sucrose, and sectioned (10-40 $\mu \mathrm{m})$.

Immunofluorescence. Immunostaining was performed as previously described (45). Confocal imaging was performed on an SP5 Leica confocal microscope. Antibodies were against $\alpha$ SMA (Abcam, ab5694), cleaved caspase-3 (Ozyme, 9661), PECAM1 (BD, clone MEC 13.3), CD44 (BD, clone IM7), CD45 (eBioscience, clone 30F11), CD146 (eBioscience, clone P1H12), collagen type I (Southern Biotech, 1310), FSP1 (Abcam, ab27957), PDGFR $\alpha$ (R\&D Systems, AF1062), PDGFR $\beta$ (46), VE-cadherin (BD Biosciences, clone 11D4.1), Vimentin (Epitomics, 4551), Wt1 (Santa Cruz, sc-192), and Tie2 (eBioscience, clone TEK4). Quantification of VE-cadherin-CreERT2 recombination and cell counting were performed using Volocity Image Analysis software.

Trichrome staining. Trichrome staining was performed using a Trichrome Stain (Masson) Kit (HT15, Sigma-Aldrich), as specified by the manufacturer.

Flow cytometry/cell sorting. Hearts were isolated and perfused briefly with HBSS in order to remove blood. Hearts were then perfused with collagenase/ dispase (Roche), and the LVs/septums were removed. After removing the valves, hearts were minced and dissociated in collagenase type II (Worthington) and resuspended in HBSS with 2\% to 3\% FBS, $2.5 \mathrm{mM}$ EDTA. The cell suspension was subjected to FACS analysis/sorting using a BD FACSCalibur II/Aria. The following antibodies were used: antiCD31-APC (eBioscience, clone 390), anti-CD45-PECy7/Brilliant Violet (eBioscience/Biolegend, clone 30F11), anti-PDGFR $\alpha$-APC (eBioscience, clone APA5), and anti-Thy1 (eBioscience, clone 53-2.1). DAPI was used as a viability dye. Data were analyzed using FlowJo software.

Tamoxifen inductions. Tamoxifen (Sigma-Aldrich) was prepared according to the manufacturer's instructions. For embryonic studies, a single i.p. injection was administered to the mother $(70 \mathrm{mg} / \mathrm{kg} \mathrm{BW})$. For labeling of adult endothelium, 5 consecutive daily i.p. injections were administered $(70 \mathrm{mg} / \mathrm{kg} \mathrm{BW} / \mathrm{d})$. For labeling of adult epicardium, adult males were administered 3 consecutive daily i.p. injections $(70 \mathrm{mg} / \mathrm{kg} \mathrm{BW} / \mathrm{d})$.

TAC. Ascending aortic constriction was performed on 8- to 10-week-old male mice as previously described (47). Echocardiography and invasive hemodynamics were performed after 7, 14, and 28 days, before mice were sacrificed. For sham-operated animals, the chest was opened but no banding was performed.

Proliferation assay. Mice received a single i.p. injection of EdU (Invitrogen) $(20 \mathrm{mg} / \mathrm{kg} \mathrm{BW}) 72$ hours after surgery, and hearts were recovered 24 hours later and processed according to the manufacturer's instructions.

$R N A$ isolation and $q R T-P C R$. For whole tissue, Trizol extraction was used according to the manufacturer's instructions. For sorted cells, the Zymo Quick-RNA MicroPrep was used according to the manufacturer's instructions. First-strand synthesis, RT-PCR reactions and primer design were carried out as previously described (45). Briefly, first-strand cDNA was generated using random hexamers and the Invitrogen Superscript III Kit (Invitrogen). Primers for RT-PCR, listed as follows, or previously published (45), were designed using Primer3 software (http:// primer3.wi.mit.edu) and optimized using control murine cDNA: fsp1: 5'-AGAGGCAGGCTCAGCAAAT-3', 5'-CAGTTAAAACCCCGGCTTC-3'; pdgfro: 5'-ACCTCCCACCAGGTCTTTCT-3', 5'-CTCATTCTCGTTTGGGAGGA-3'; cdh5 (Vecad): 5'-TGAGGCAATCAACTGTGCTC-3', 5'-TTCGTGGAGGAGCTGATCTT-3'; postn: 5'-AACGTCTGTGCCCTCCAG-3', 5'-AGCCTTTCATCCCTTCCATT-3'; ddr2: 5'-GAAATCTACAAGCGACCTGACAT-3', 5'-GATCATTTCAGAAGACGGAGTTG-3'; col11a1: 5'-AAAACGAAACGGTGGATCTG-3', 5'-TGTTTGTGCAAAATCCTGTTG-3'; wisp1: 5'-TCCTGTGACGCTGACTTCC-3' ' 5'-AGAATTCGGGGCGTGTAGT-3'; P4h: 5'-GGATATGAGCGGAAAGTCCA-3', 5'-AGGATCTGGCTTCGCTGTAA-3'. RT-PCR reactions were performed using 96-well low-profile PCR plates in the CFX96 Bio-Rad thermocycler (Bio-Rad).
Echocardiographic analysis. Transthoracic echocardiography was performed on mice under the conditions listed below. Here, mice were anesthetized via $1 \%$ isoflurane and moved to a biofeedback warming station that maintained core body temperature. Mice were then placed under anesthesia ( $0.5 \%$ isoflurane). Ultrasound gel was applied to the chest of the animal, and echocardiography measurements were obtained using the VEVO 2100 software package and ultrasound system with a linear transducer, with a $32-55 \mathrm{MHz}$ operating frequency (Visual Sonics, SonoSite). M-mode tracings were taken and measured for wall thicknesses and interdimensional space for both systole and diastole of the heart. Ejection fraction and fractional shortening were calculated as previously described (45).

Hemodynamic measurements. At 4, 7, 14, and 28 days, hemodynamic measurements were performed as described previously (47).

Microarrays and clustering analyses. Affymetrix 430 v2 microarrays were hybridized to cRNA prepared from RNA isolated with the Zymo QuickRNA MicroPrep Kit and using NuGEN Ovation Pico WTA System V2 according to manufacturer's instructions. RNA was prepared from sorted cells pooled from 3 mice per condition. Affymetrix expression signal values were generated and normalized with RMA using AltAnalyze (48). Hierarchical ordered partitioning and collapsing hybrid (HOPACH) clustering was used to determine all possible pairwise correlations as previously described (49). Differentially expressed probe sets (19,936 probe sets corresponding to 8,752 unique NCBI gene IDs displayed a $\mid$ fold $\mid>2$ in at least one group compared with mean expression across all groups) were clustered with average uncentered hierarchical clustering using Eisen cluster (50). This corresponds to 8,752 unique NCBI gene IDs. Gene set enrichment analysis was conducted with GO-Elite (51). Array data can be accessed at GEO using accession number GSE45820.

Statistics. Data are presented as mean \pm SEM unless indicated otherwise. We used 2-tailed Student's $t$ test or ANOVA for comparisons among groups as indicated. Analysis was performed using GraphPad Prism software. $P$ values of less than 0.05 were considered significant. Healthy male animals were selected based on genotype and were randomly allocated to sham/TAC groups for pressure overload studies.

Study approval. All animal procedures were approved by the UCSD Animal Care and Use Committee. UCSD has an Animal Welfare Assurance (A3033-01) on file with the Office of Laboratory Animal Welfare and is fully accredited by AAALAC International.

\section{Acknowledgments}

S.M. Evans is funded by grants from the National Heart, Lung, and Blood Institute (HL-117649 and HL119967). J. Chen is funded by grants from the National Heart, Lung, and Blood Institute (HL066100 and HL106968). J. Chen is the American Heart Association (AHA) Endowed Chair in Cardiovascular Research. T. Moore-Morris and I. Banerjee are supported by AHA postdoctoral fellowships (11POST7310066 and 12POST12030256). Microscopy work was performed at the UCSD Neuroscience Microscopy Shared Facility and was supported by the National Institutes of Health (grant P30 NS047101). Microarrays were performed at the UCLA Clinical Microarray Core.

Received for publication December 26, 2013, and accepted in revised form April 24, 2014.

Address correspondence to: Sylvia Evans, University of California, San Diego, School of Medicine, Basic Science Building Room 5023, La Jolla, California 92130, USA. Phone: 858.822.2452; Fax: 858.822.3027; E-mail: syevans@ucsd.edu. 
1. Lloyd-Jones D, et al. Heart disease and stroke statistics - 2010 update: a report from the American Heart Association. Circulation. 2010;121(7):e46-e215.

2. Heidenreich PA, et al. Forecasting the future of cardiovascular disease in the United States: a policy statement from the American Heart Association. Circulation. 2011;123(8):933-944.

3. Banerjee I, Yekkala K, Borg TK, Baudino TA. Dynamic interactions between myocytes, fibroblasts, and extracellular matrix. Ann N Y Acad Sci. 2006;1080:76-84.

4. Mikawa T, Gourdie RG. Pericardial mesoderm generates a population of coronary smooth muscle cells migrating into the heart along with ingrowth of the epicardial organ. Dev Biol. 1996;174(2):221-232.

5. Cai CL, et al. A myocardial lineage derives from Tbx18 epicardial cells. Nature. 2008;454(7200):104-108.

6. Zeisberg EM, et al. Endothelial-to-mesenchymal transition contributes to cardiac fibrosis. Nat Med. 2007;13(8):952-961.

7. Widyantoro B, et al. Endothelial cell-derived endothelin-1 promotes cardiac fibrosis in diabetic hearts through stimulation of endothelial-to-mesenchymal transition. Circulation. 2010;121(22):2407-2418.

8. van Amerongen MJ, et al. Bone marrow-derived myofibroblasts contribute functionally to scar formation after myocardial infarction. $J$ Pathol. 2008;214(3):377-386

9. Zhou B, et al. Adult mouse epicardium modulates myocardial injury by secreting paracrine factors. J Clin Invest. 2011;121(5):1894-1904.

10. Osterreicher $\mathrm{CH}$, et al. Fibroblast-specific protein 1 identifies an inflammatory subpopulation of macrophages in the liver. Proc Natl Acad Sci U S A. 2011;108(1):308-313.

11. Kong P, Christia P, Saxena A, Su Y, Frangogiannis NG. Lack of specificity of fibroblast-specific protein 1 in cardiac remodeling and fibrosis. Am J Physiol Heart Circ Physiol. 2013;305(9):H1363-H1372.

12. Yata Y, et al. DNase I-hypersensitive sites enhance alpha1(I) collagen gene expression in hepatic stellate cells. Hepatology. 2003;37(2):267-276.

13. Zeisberg EM, Kalluri R. Origins of cardiac fibroblasts. Circ Res. 2010;107(11):1304-1312.

14. Smith CL, Baek ST, Sung CY, Tallquist MD. Epicardial-derived cell epithelial-to-mesenchymal transition and fate specification require PDGF receptor signaling. Circ Res. 2011;108(12):e15-e26.

15. Rudat C, Norden J, Taketo MM, Kispert A. Epicardial function of canonical Wnt-, Hedgehog-, Fgfr1/2-, and Pdgfra-signaling. Cardiovasc Res. 2013;100(3):411-421.

16. Koumas L, King AE, Critchley HO, Kelly RW, Phipps RP. Fibroblast heterogeneity: existence of functionally distinct Thy $1(+)$ and Thy $1(-)$ human female reproductive tract fibroblasts. Am J Pathol. 2001;159(3):925-935

17. Sirish $\mathrm{P}$, et al. Unique mechanistic insights into the beneficial effects of soluble epoxide hydrolase inhibitors in the prevention of cardiac fibrosis. Proc Natl Acad Sci U S A. 2013;110(14):5618-5623.

18. Rockman HA, et al. Segregation of atrial-specific and inducible expression of an atrial natriuretic factor transgene in an in vivo murine model of cardiac hypertrophy. Proc Natl Acad Sci U S A. 1991;88(18):8277-8281

19. Wessels A, et al. Epicardially derived fibroblasts preferentially contribute to the parietal leaflets of the atrioventricular valves in the murine heart. Dev Biol. 2012;366(2):111-124

20. Madisen $\mathrm{L}$, et al. A robust and high-throughput Cre reporting and characterization system for the whole mouse brain. Nat Neurosci. 2010;13(1):133-140.

21. Zhou B, et al. Epicardial progenitors contribute to the cardiomyocyte lineage in the developing heart. Nature. 2008;454(7200):109-113.

22. de Lange FJ, et al. Lineage and morphogenetic analysis of the cardiac valves. Circ Res. 2004;95(6):645-654.

23. de Vlaming A, et al. Atrioventricular valve development: new perspectives on an old theme. Differentiation. 2012;84(1):103-116.

24. van Nierop BJ, et al. Phenotyping of left and right ventricular function in mouse models of compensated hypertrophy and heart failure with cardiac MRI. PLoS One. 2013;8(2):e55424.

25. Bochmann L, Sarathchandra P, Mori F, Lara-Pezzi E, Lazzaro D, Rosenthal N. Revealing new mouse epicardial cell markers through transcriptomics. PLoS One. 2010;5(6):e11429.

26. Red-Horse K, Ueno H, Weissman IL, Krasnow MA. Coronary arteries form by developmental reprogramming of venous cells. Nature. 2010; 464(7288):549-553

27. Wu B, et al. Endocardial cells form the coronary arteries by angiogenesis through myocardial-endocardial VEGF signaling. Cell. 2012;151(5):1083-1096.

28. Lincoln J, Alfieri CM, Yutzey KE. Development of heart valve leaflets and supporting apparatus in chicken and mouse embryos. Dev Dyn. 2004;230(2):239-250.

29. Gustafsson E, Brakebusch C, Hietanen K, Fassler $\mathrm{R}$. Tie-1-directed expression of Cre recombinase in endothelial cells of embryoid bodies and transgenic mice. J Cell Sci. 2001;114(pt 4):671-676

30. Kriz W, Kaissling B, Le Hir M. Epithelial-mesenchymal transition (EMT) in kidney fibrosis: fact or fantasy? J Clin Invest. 2011;121(2):468-474.

31. Ieronimakis $\mathrm{N}$, et al. Coronary adventitial cells are linked to perivascular cardiac fibrosis via TGF $\beta 1$ signaling in the $\mathrm{mdx}$ mouse model of Duchenne muscular dystrophy. J Mol Cell Cardiol. 2013;63:122-134

32. Tomasek JJ, Gabbiani G, Hinz B, Chaponnier C, Brown RA. Myofibroblasts and mechano-regulation of connective tissue remodelling. Nat Rev Mol Cell Biol. 2002;3(5):349-363.

33. Rudat C, Kispert A. Wt1 and epicardial fate mapping. Circ Res. 2012;111(2):165-169.

34. Katz TC, et al. Distinct compartments of the proepicardial organ give rise to coronary vascular endothelial cells. Dev Cell. 2012;22(3):639-650.

35. Iredale JP, et al. Mechanisms of spontaneous resolution of rat liver fibrosis. Hepatic stellate cell apoptosis and reduced hepatic expression of metalloproteinase inhibitors. J Clin Invest. 1998;102(3):538-549.

36. Mollmann $\mathrm{H}$, et al. Bone marrow-derived cells contribute to infarct remodelling. Cardiovasc Res. 2006;71(4):661-671

37. Asahara T, et al. Bone marrow origin of endothelial progenitor cells responsible for postnatal vasculogenesis in physiological and pathological neovascularization. Circ Res. 1999;85(3):221-228.

38. Takahashi $\mathrm{T}$, et al. Ischemia- and cytokine-induced mobilization of bone marrow-derived endothelial progenitor cells for neovascularization. Nat Med. 1999;5(4):434-438.

39. Bucala R, Spiegel LA, Chesney J, Hogan M, Cerami A. Circulating fibrocytes define a new leukocyte subpopulation that mediates tissue repair. $\mathrm{Mol}$ Med. 1994;1(1):71-81.

40. Braitsch CM, Kanisicak O, van Berlo JH, Molkentin JD, Yutzey KE. Differential expression of embryonic epicardial progenitor markers and localization of cardiac fibrosis in adult ischemic injury and hypertensive heart disease. J Mol Cell Cardiol. 2013;65:108-119.

41. Teekakirikul P, et al. Cardiac fibrosis in mice with hypertrophic cardiomyopathy is mediated by nonmyocyte proliferation and requires Tgf- $\beta$. J Clin Invest. 2010;120(10):3520-3529.

42. Kisanuki YY, Hammer RE, Miyazaki J, Williams SC, Richardson JA, Yanagisawa M. Tie2-Cre transgenic mice: a new model for endothelial cell-lineage analysis in vivo. Dev Biol. 2001;230(2):230-242.

43. Stadtfeld M, Graf T. Assessing the role of hematopoietic plasticity for endothelial and hepatocyte development by non-invasive lineage tracing. Development. 2005;132(1):203-213.

44. Monvoisin A, Alva JA, Hofmann JJ, Zovein AC, Lane TF, Iruela-Arispe ML. VE-cadherin-CreERT2 transgenic mouse: a model for inducible recombination in the endothelium. Dev Dyn. 2006;235(12):3413-3422.

45. Banerjee I, et al. Thymosin $\beta 4$ is dispensable for murine cardiac development and function. Circ Res. 2012;110(3):456-464.

46. Ozerdem U, Grako KA, Dahlin-Huppe K, Monosov E, Stallcup WB. NG2 proteoglycan is expressed exclusively by mural cells during vascular morphogenesis. Dev Dyn. 2001;222(2):218-227.

47. Sheikh F, et al. An FHL1-containing complex within the cardiomyocyte sarcomere mediates hypertrophic biomechanical stress responses in mice. J Clin Invest. 2008;118(12):3870-3880.

48. Salomonis N, et al. Alternative splicing regulates mouse embryonic stem cell pluripotency and differentiation. Proc Natl Acad Sci U S A. 2010; 107(23):10514-10519.

49. Felfly H, Xue J, Zambon AC, Muotri A, Zhou D, Haddad GG. Identification of a neuronal gene expression signature: role of cell cycle arrest in murine neuronal differentiation in vitro. Am J Physiol Regul Integr Comp Physiol. 2011; 301(3):R727-R745

50. Eisen MB, Spellman PT, Brown PO, Botstein D. Cluster analysis and display of genome-wide expression patterns. Proc Natl Acad Sci U S A. 1998; 95(25):14863-14868.

51. Zambon AC, et al. GO-Elite: a flexible solution for pathway and ontology over-representation. Bioinformatics. 2012;28(16):2209-2210. 